\title{
Formation Control of Unmanned Surface Vehicles with Sensing Constraints Using Exponential Remapping Method
}

\author{
Jian Wang, ${ }^{1,2}$ Jing-Yang Liu, ${ }^{1,2}$ and Hong $\mathrm{Yi}^{1,2}$ \\ ${ }^{1}$ State Key Laboratory of Ocean Engineering, Shanghai Jiao Tong University, Shanghai 200240, China \\ ${ }^{2}$ Collaborative Innovation Center for Advanced Ship and Deep-Sea Exploration, Shanghai Jiao Tong University, \\ Shanghai 200240, China \\ Correspondence should be addressed to Jing-Yang Liu; jy_liu@sjtu.edu.cn
}

Received 18 May 2017; Revised 6 August 2017; Accepted 14 August 2017; Published 13 September 2017

Academic Editor: Jean Jacques Loiseau

Copyright (C) 2017 Jian Wang et al. This is an open access article distributed under the Creative Commons Attribution License, which permits unrestricted use, distribution, and reproduction in any medium, provided the original work is properly cited.

\begin{abstract}
This paper presents a formation control strategy for unmanned surface vehicles (USVs) with sensing constraints moving in a leaderfollower formation. Each USV is assumed to be equipped with a vision-based sensor, which is able to get the line-of-sight (LOS) range and bearing information. Most existing literature assumes that the USV s in formation control are with no sensing limitations or with 360-degree sensing fields; however, in our research, the vision-based sensor's capability is restricted due to limited Field of View (FOV) and visual range. We consider that each USV in formation problem is equipped with a sector-like sensing field sensor for the leader-follower formation in two-dimensional space. The formation controller is developed by employing backstepping control technique and exponential remapping. The backstepping controller is designed to stabilize the triangular formation of three USVs, and the proposed exponential remapping method is to deal with the sector-like sensing constraint problem. Comparative analysis with three exponential remapping methods using numerical simulations is given to demonstrate the effectiveness of the proposed method.
\end{abstract}

\section{Introduction}

Over the past few years, due to the increasing need for utilizing multiple vehicles to perform difficult tasks as a team, the formation control of multivehicle systems has gained enormous interests in the research of system and control. Maintaining a desired formation enables multiple vehicles to cooperate with each other to accomplish some difficult tasks which are not executable by a single one. And it can reduce system cost, enhance work efficiency, and provide redundancy against individual failures [1]. The relevant applications have arisen in marine engineering including collaborative antisubmarine, costal patrols, environmental monitoring, disaster search, and underway ship replenishment, to name just a few. Many researchers have made contributions to the formation control, and several methods have been proposed including leader-follower method [1-5], virtual structure strategy $[6,7]$, behavior-based approach $[8,9]$, graph theorybased method [10-12], and artificial potential method [13].
Leader-follower $(L-F)$ method is most preferred in practice because of its simplicity and scalability [3]. In a leaderfollower formation, one or more individual of the group is designated as the leader, and the others are designated as followers; the followers follow the leader to keep the desired distances or angles; then the formation will be achieved ultimately. During the last decade, the formation control has been focused on the marine vehicles, and most works appear to have been done within the leader-follower framework. A method for formation control of marine surface craft inspired by Lagrangian mechanics was presented in [14], and the method is also suitable for underactuated ships. In [15], within a leader-follower framework, fully actuated ships were controlled as a formation by applying the integrator backstepping and cascade theory. In [16], the problem of path following and formation control for underactuated surface vessels in the presence of unknown ocean currents was considered, and the proposed controllers were based on line-of-sight (LOS) guidance, adaptive control, and cascaded systems 
theory. In [4], formation control of multiple underactuated autonomous underwater vehicles (AUVs) was concerned, where a virtual vehicle was constructed such that its trajectory converged to the reference trajectory of the follower. Also, using a nonlinear observer to estimate the leader's velocity and acceleration, a leader-follower output synchronization control scheme was developed in [17] for ship replenishment operations. In [18], a new implementable cooperative adaptive backstepping controller was proposed for a group of AUVs to track a time-varying virtual leader, where the singular perturbation theory was also used so that the velocity information of the neighbor was precluded in the cooperative design. In [19], the neural network and approach angle were used to implement formation control of AUVs without the velocity information of the leader. In general, among these control designs, two types of their information exchange methods can be summarized. One type for $L-F$ formation control has wireless communication between leaders and followers, and the main issues they need to handle are communication delay, packet lose [20,21], and so forth. The other type for $L-F$ formation control requires the local sensors such as radar or sonar to measure the vehicles' states and copes with noises which are bad for measurements, such that the robots in $[2,22]$ were equipped with omnidirectional cameras, which had 360-degree views of surroundings. In [5], each unicycle robot was equipped with a panoramic camera that only provides the view angle to the other robots. In [23], a constructive method was proposed to force a group of multimobile robots to stabilize at a desired location, where the robots were with limited sensing ranges. A robust adaptive formation controller was developed by employing neural network and dynamic surface control technique to keep the underactuated autonomous surface vehicles (ASVs) moving in an $L-F$ formation in [1], where the proposed method only used the measurements of LOS range and angle by local sensors. Robots in [24] could measure the relative positions of any object if the object was within a given sensing distance and there were no obstacles in between. The author in [25] presented a constructive method to design cooperative controllers that forced a group of several underactuated ships with limited sensing ranges to perform a desired formation and guaranteed no collisions between the ships. The agents considered in most of these existing works are assumed to be without sensing limitations or with 360 -degree sensing fields. However, in practice, it is inevitable that there are some sensing constraints on sensing fields, for example, Lidar and camera. Lidar is widely used on robots and unmanned ground vehicles; however, some Lidars can only scan a sector area in a special plane. Cameras are also frequently used onboard sensors; most of them (except for the omnidirectional camera) have the cone-like sensing fields. Some attempts have been made to solve the similar problem about sensing constraints. In [26], a leader-follower formation control strategy was presented for underactuated marine vehicles which moved under sensing and communication constraints in the presence of bounded persistent environmental disturbances, and the sensing area was limited as a circular sector. The proposed control design guarantees that the follower always keeps sensing with the leader. However the follower is centered in the camera field, which is inapplicable for two or more followers to obtain a triangular formation. A shortest path algorithm was proposed for a robot with unicycle kinematics to go to a goal position in [27], and an onboard camera with limited Field of View (FOV) was used. The algorithm is able to make the robot follow shortest paths to its goal position while keeping a given feature in sight. Frequent turning, going forward or backward, is required, which is easy to do for unicycle robots, but it is not practical for USVs. In [28], the author presented a novel navigation method for a nonholonomic robot based on Model Predictive Control (MPC). The method can keep sensing with a stationary target although there exist FOV sensing constraints. However the algorithm pays close attention to maintain the target in its sensing center, and it cannot be used directly for leaderfollower formation control.

In this study, we focus on the formation control of USVs considering sensing field constraints. Each USV is assumed to be equipped with a vision-based sensor, which is able to get the LOS range and bearing information of its leader. And each sensor has a cone-like sensing field just like a camera. Some related research like vision-based target tracking of USVs have been done by some researchers, such as in [29]; the relative velocity and position of the USV's target were estimated using calibrated projective camera model. In [30], an approach to detect and track long distance boat with high resolution image was proposed for USVs. In [31], a robust computer vision system for an USV was developed to track a moving marine vehicle from the USV. In [32], autonomous detection and tracking of a surface ship using a monocular camera mounted on an USV were addressed, and the bearing and range to the target ship with respect to the USV were obtained by computer vision techniques. On the basis of these studies, the follower can be able to get the leader's information. In this research, how the on-board vision-based sensor obtains the target's information is not the main attention; we assume that the follower can be able to get its leader's information clearly. For efficiency and reality, we consider the formation control with the leader-follower structure in two-dimensional (2D) space; that is to say, the on-board sensor's cone-like sensing field is simplified as a sector one. Because of the leader-follower structure and the sector-like sensing field, two priority rules are considered to hold by USVs in this research. First, the follower should be able to keep sensing with the leader(s) so that it can always get the information of the leader(s) and the formation will be maintained. Second, on the basis of followers keeping sensing with the leader(s), the control strategy should be able to get one optimal formation structure. In our previous work [33], we have made an effort on the formation control of unmanned surface vehicles with vision sensor constraints. However, in this work, when the follower's on-border sensor loses the information of its leader, the switch control will then work to control the follower to turn its yaw to try to find the leader again, which will not keep the information of the leader and the formation of the USVs will not be guaranteed. So in this paper, we design a formation controller by employing backstepping control technique and exponential remapping method, under which the follower can be able to keep sensing 
with the leader(s) so as to maintain the formation. The backstepping controller is designed to stabilize the triangular formation of three USVs, and an exponential remapping method is proposed to deal with the sector-like sensing constraint problem.

The contribution of this paper is twofold:

(i) In contrast to the leader-follower formation control problems in $[1-5,16-18]$, this paper addresses sensor constraint problems of leader-follower formation control of USVs. Unlike the agents without sensing limitations or with 360-degree sensing fields considered in $[2,5,22-25]$, the proposed control scheme based on backstepping control technique and exponential remapping method enables the USVs with sector-like sensing constraints to form a stable formation.

(ii) The contributions in $[26,28]$ are developing the formation controllers for USVs under leader-follower structure considering sector-like sensing constraints, and the follower keeps its target in the center of its center, which is hard to form a triangle formation. The contributions in [31] are developing the shortest path control for a robot with FOV constraints to reach a target. In contrast to the contributions of the previous work, the control scheme in this paper can form a triangle formation under sector-like sensing constraints and at the same time get the optimal formation structure.

The remainder of the paper is organized as follows: in Section 2, the modeling of the system is provided and the sensing constraints of FOV are stated. Section 3 shows the proposed formation control of the system. In Section 4, simulation results are shown. Concluding remarks are given in Section 5.

\section{Preliminary}

2.1. Kinematics and Dynamics of USV. The general 6-DOF can be decoupled to surge/forward, sway/lateral, and heading/yaw pairs, while the dynamics associated with the motion in roll, pitch, and heave are generally neglected. Assuming that the inertia, added mass, and hydrodynamic damping matrices are diagonal, which holds good for ships having port/starboard and fore/aft symmetry [34], the kinematics and dynamics of the USV with no external disturbances can be described as in [35] by

$$
\begin{aligned}
& \dot{x}=u \cos (\varphi)-v \sin (\varphi) \\
& \dot{y}=u \sin (\varphi)+v \cos (\varphi) \\
& \dot{\varphi}=r \\
& \dot{u}=\frac{m_{2}}{m_{1}} v r-\frac{d_{1}}{m_{1}} u+\frac{1}{m_{1}} \tau_{u} \\
& \dot{v}=-\frac{m_{1}}{m_{2}} u r-\frac{d_{2}}{m_{2}} v \\
& \dot{r}=\frac{m_{1}-m_{2}}{m_{3}} u v-\frac{d_{3}}{m_{3}} r+\frac{1}{m_{3}} \tau_{r} .
\end{aligned}
$$

Here, $(x, y)$ denotes the position and $\varphi$ is the heading angle of the USV in the earth-fixed frame; $u, v$, and $r$ are the velocities of the USV in the body-fixed frame ( $u$ : surge velocity; $v$ : sway velocity; $r$ : angular velocity in yaw); $m_{i}(i=$ $1,2,3)$ is the inertia of the USV including added masses; $d_{i}(i=1,2,3)$ is the hydrodynamic damping in surge, sway, and yaw; $\tau_{u}$ and $\tau_{r}$ are the control signal ( $\tau_{u}$ : surge force; $\tau_{r}$ : yaw moment). For the actual USV, the velocities and input forces are limited, so we make the following assumption.

Assumption 1. The velocities and input forces of the USV are bounded as $\sup _{t}\|u\|=\bar{u}_{\max }, \sup _{t}\|v\|=\bar{v}_{\max }, \sup _{t}\|r\|=\bar{r}_{\max }$, $\sup _{t}\left\|\tau_{u}\right\|=\bar{\tau}_{u \max }$, and $\sup _{t}\left\|\tau_{r}\right\|=\bar{\tau}_{r \max }$ with known bounds $\bar{u}_{\text {max }}>0, \bar{v}_{\text {max }}>0, \bar{r}_{\text {max }}>0, \bar{\tau}_{u \max }>0$, and $\bar{\tau}_{\text {rmax }}>0$.

2.2. Leader-Follower Formation. In this paper, the leader vehicle will be denoted with a subscript " $L$," while the $i$ th follower vehicle will be denoted with a subscript " $F i$." We use the Cartesian coordinates rather than the commonly used polar coordinates because of the models having singularity problem in the polar coordinate representation as shown in [36]. Figure 1 presents the basic geometric structure of two USVs moving in the leader-follower formation in Cartesian coordinates, where $\left(X_{L}, Y_{L}, \varphi_{L}\right)$ are the position and yaw angle of the leader, $\left(X_{F i}, Y_{F i}, \varphi_{F i}\right)$ are the position and yaw angle of the follower $i$, and $\left(l_{L F i}, \alpha_{L i}\right)$ denotes the LOS range and bearing information between the leader and the follower $i$. And $\lambda_{L F i}$ is the relative angle with respect to the leader.

The formation is defined as $\mathbb{F}_{L F i}=\left[\begin{array}{ll}l_{L F i} & \lambda_{L F i}\end{array}\right]^{T}$, where $l_{L F i} \in \mathbb{R} \geq N_{D}, \lambda_{L F i} \in(\pi / 2,3 \pi / 2) . N_{D}$ denotes the minimum distance between leader and followers in case collisions between them. The desired formation is defined as $\mathbb{F}_{L F i}^{d}=$ $\left[\begin{array}{ll}l_{L F i}^{d} & \lambda_{L F i}^{d}\end{array}\right]^{T}$. Given the leader's position and yaw angle, and as long as $\mathbb{F}_{L F i}$ is known and fixed, the follower's position will be unique. So the desired formation $\mathbb{F}_{L F i}^{d}$ will be maintained.

In Figure $1, l_{x}$ and $l_{y}$ are the projects of $l_{L F i}$ along $x$ and $y$ directions relative to the leader; $l_{x}^{d}$ and $l_{y}^{d}$ are the desired values of $l_{x}$ and $l_{y}$. They can be got as follows:

$$
\begin{aligned}
& l_{x}=-\left(X_{L}-X_{F}\right) \cos \varphi_{L}-\left(Y_{L}-Y_{F}\right) \sin \varphi_{L} \\
& l_{y}=\left(X_{L}-X_{F}\right) \sin \varphi_{L}-\left(Y_{L}-Y_{F}\right) \cos \varphi_{L}, \\
& l_{x}^{d}=l_{L F i}^{d} \sin \left(\lambda_{L F i}^{d}+\varphi_{L}-\frac{\pi}{2}\right), \\
& l_{y}^{d}=l_{L F i}^{d} \cos \left(\lambda_{L F i}^{d}+\varphi_{L}-\frac{\pi}{2}\right) .
\end{aligned}
$$

Then combining (1) and (3), we can obtain the following kinematic equations:

$$
\begin{aligned}
& \dot{l}_{x}=u_{L}-u_{F} \cos e_{\varphi}-v_{F} \sin e_{\varphi}+l_{y} \dot{\varphi}_{L} \\
& \dot{l}_{y}=v_{L}+u_{F} \sin e_{\varphi}-v_{F} \cos e_{\varphi}-l_{x} \dot{\varphi}_{L} \\
& \dot{e}_{\varphi}=\dot{\varphi}_{L}-\dot{\varphi}_{F}=r_{L}-r_{F} .
\end{aligned}
$$




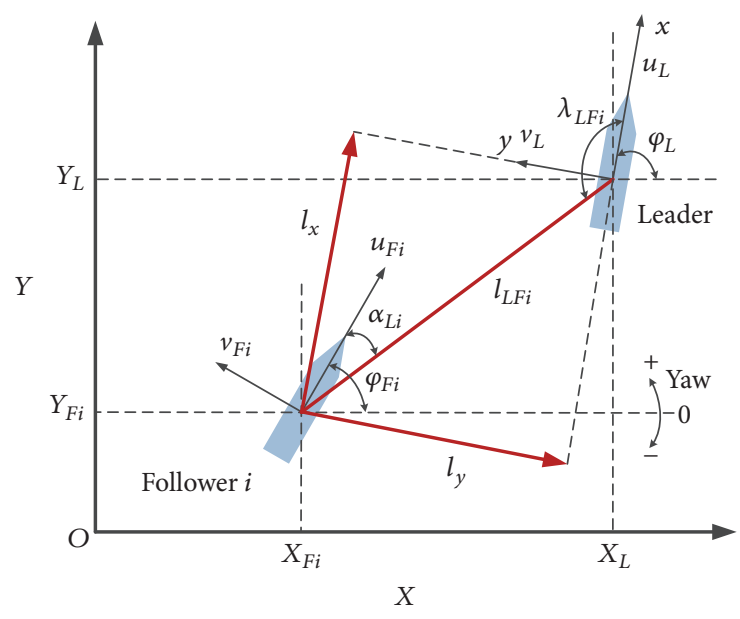

FIGURE 1: Leader-follower formation.

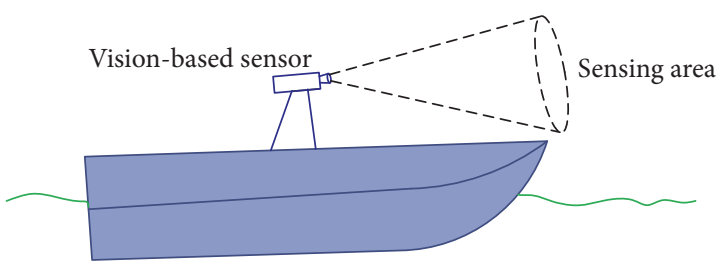

FIGURE 2: USV equipped with a vision-based sensor.

The control objective is to design the formation controller for the follower that follows the leader keeping the desired values $l_{x}^{d}, l_{y}^{d}$, and $e_{\varphi}$ stable.

2.3. Sensing Constraints. In this paper, each USV is assumed to be equipped with a vision-based sensor, which is able to get the LOS range and bearing information of its sensing object. The vision-based sensor's capability is restricted due to limited FOV and visual range, as shown in Figure 2. As mentioned in Section 1, we consider the formation control with the leader-follower structure in two-dimensional (2D) space; that is to say, the on-board sensor's sensing field is simplified as a sector-like one, as shown in Figure 3.

The circular sector of radius $D_{\text {range }}$ and angle $2 \alpha$ in Figure 3 define the effective sensing field of each USV. Thus each vehicle can get the measurements of the objects moving within its sensing field. $D_{\text {range }}$ and $2 \alpha$ are the maximum sensing range and maximum sensing angle of the on-board vision-based sensor. $\alpha_{L}$ and $l_{L-F i}$ denote the leader's orientation and distance relative to the follower $i$ measured by the follower $i$ 's on-board vision-based sensor; that is to say, they are the follower's sensing angle and distance. The two USVs shown in Figure 3 can form a formation if and only if the follower $i$ maintains sensing with the leader. To facilitate the control design, we make the following assumptions.

Assumption 2. The formation state vector $\mathbb{F}_{L F i}$ can be measured accurately by the follower's on-board sensor. And the

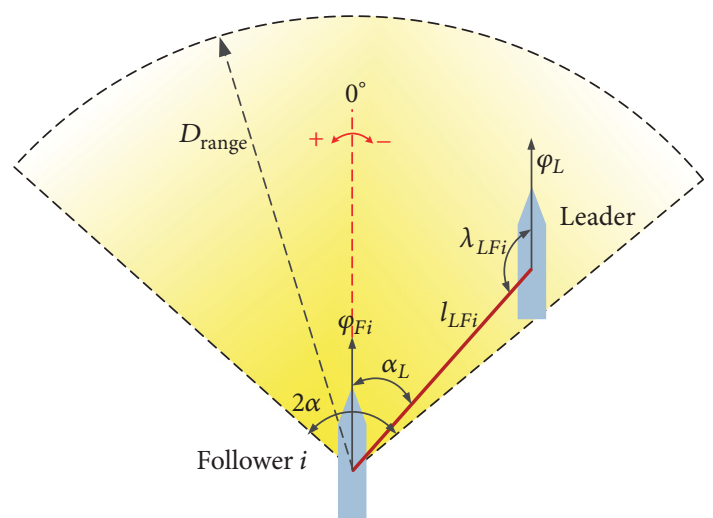

Figure 3: Sector-like sensing field.

leader's velocities, acceleration, and orientation are assumed to be able to be measured and calculated by the follower.

Assumption 3. The FOV of the on-board sensor has a constraint angle $2 \alpha$. The sensing range of the sensor $d_{v}$ is bounded as $\sup \left(d_{v}\right)=D_{\text {range }}$. And the desired range $l_{L F i}^{d}$ between leader and follower is bounded as $\sup \left(l_{L F i}^{d}\right) \ll D_{\text {range }}$.

Assumption 4. The single USV can be able to obtain its orientation and velocities data accurately from its on-board sensors. No disturbances and obstacles are considered in this formation system.

\section{Control Strategy}

In this section, we will apply the backstepping control method and exponential remapping method to design the torque controls $\tau_{u}$ and $\tau_{r}$ to achieve the formation control. In the first step, virtual controls are designed using formation errors. In the second step, actual controls $\tau_{u}$ and $\tau_{r}$ are designed. At last, considering the sensing constraints, position errors, and yaw angle errors are remapped using exponential remapping method.

3.1. Virtual Controls. Define the formation position errors in $x$ and $y$ directions as

$$
E=\left[\begin{array}{l}
e_{x} \\
e_{y}
\end{array}\right]=\left[\begin{array}{l}
l_{x}-l_{x}^{d} \\
l_{y}-l_{y}^{d}
\end{array}\right] .
$$

Combining (4), (5), and (6), then the error dynamics of the system are given as

$$
\begin{aligned}
& {\left[\begin{array}{c}
\dot{e}_{x} \\
\dot{e}_{y} \\
\dot{e}_{\varphi}
\end{array}\right]=\left[\begin{array}{c}
j_{x}-j_{x}^{d} \\
\dot{l}_{y}-j_{y}^{d} \\
\dot{\varphi}_{L}-\dot{\varphi}_{F}
\end{array}\right]} \\
& =\left[\begin{array}{c}
-u_{F} \cos e_{\varphi}-v_{F} \sin e_{\varphi}+e_{y} \dot{\varphi}_{L}+u_{L}+l_{y}^{d} \dot{\varphi}_{L}-j_{x}^{d} \\
u_{F} \sin e_{\varphi}-v_{F} \cos e_{\varphi}-e_{x} \dot{\varphi}_{L}+v_{L}-l_{x}^{d} \dot{\varphi}_{L}-\dot{l}_{y}^{d} \\
r_{L}-r_{F}
\end{array}\right] .
\end{aligned}
$$


The followers are the controlled objects; however, we note that the formation position error $E$ is in the leader's bodyfixed frame. So using an Euler angle rotation matrix, we can get the formation position error $\Sigma$ as

$$
\Sigma=\left[\begin{array}{l}
\varepsilon_{1} \\
\varepsilon_{2}
\end{array}\right]=\left[\begin{array}{cc}
\cos e_{\varphi} & -\sin e_{\varphi} \\
\sin e_{\varphi} & \cos e_{\varphi}
\end{array}\right] E .
$$

Then we can get

$$
\left[\begin{array}{c}
\dot{\varepsilon}_{1} \\
\dot{\varepsilon}_{2}
\end{array}\right]=\left[\begin{array}{c}
u_{F}+\xi_{1} \cos e_{\varphi}-\xi_{2} \sin e_{\varphi}+\varepsilon_{2} r_{F} \\
v_{F}+\xi_{1} \sin e_{\varphi}+\xi_{2} \cos e_{\varphi}-\varepsilon_{1} r_{F}
\end{array}\right],
$$

where $\xi_{1}=-u_{L}+l_{y}^{d} \dot{\varphi}_{L}-i_{x}^{d}, \xi_{2}=-v_{L}-l_{x}^{d} \dot{\varphi}_{L}-j_{y}^{d}$.

Step 1. Define the control Lyapunov function (CLF):

$$
V_{1}=\frac{1}{2} \varepsilon_{1}^{2}+\frac{1}{2} \varepsilon_{2}^{2},
$$

whose time derivative combined with (9) is given by

$$
\begin{aligned}
\dot{V}_{1}= & \varepsilon_{1} \dot{\varepsilon}_{1}+\varepsilon_{2} \dot{\varepsilon}_{2} \\
= & \varepsilon_{1}\left(u_{F}+\xi_{1} \cos e_{\varphi}-\xi_{2} \sin e_{\varphi}\right) \\
& +\varepsilon_{2}\left(v_{F}+\xi_{1} \sin e_{\varphi}+\xi_{2} \cos e_{\varphi}\right) .
\end{aligned}
$$

Choose the kinematics virtual control law as

$$
\begin{aligned}
& u_{F}^{v}=-k_{1} \varepsilon_{1}-\xi_{1} \cos e_{\varphi}+\xi_{2} \sin e_{\varphi}, \\
& v_{F}^{v}=-k_{2} \varepsilon_{2}-\xi_{1} \sin e_{\varphi}-\xi_{2} \cos e_{\varphi},
\end{aligned}
$$

where $k_{1}, k_{2}>0 \in \mathbb{R}$.

Then (11) becomes

$$
\dot{V}_{1}=-k_{1} \varepsilon_{1}^{2}-k_{2} \varepsilon_{2}^{2} \leq 0 .
$$

So the system $\Sigma$ is asymptotically stable.

Step 2. Define the sway velocity error as $v_{e}=v_{F}-v_{F}^{v}$, whose time derivative combined with (2) and (13) is given by

$$
\begin{aligned}
\dot{v}_{e}= & \dot{v}_{F}-\dot{v}_{F}^{v} \\
= & -\frac{d_{2}}{m_{2}} v_{F}-\frac{m_{1}}{m_{2}} k_{1} \varepsilon_{1} r_{F}+\frac{m_{1}}{m_{2}} \xi_{1} \cos e_{\varphi} r_{F} \\
& -\frac{m_{1}}{m_{2}} \xi_{2} \sin e_{\varphi} r_{F}+k_{2} v_{F}-k_{2} \xi_{1} \sin e_{\varphi} \\
& -k_{2} \xi_{2} \cos e_{\varphi}-k_{2} \varepsilon_{1} r_{F}-\dot{\xi}_{1} \sin e_{\varphi}-\dot{\xi}_{2} \cos e_{\varphi} \\
& -\xi_{1} \cos e_{\varphi} \dot{e}_{\varphi}+\xi_{2} \sin e_{\varphi} \dot{e}_{\varphi} .
\end{aligned}
$$

Choosing $k_{1}=d_{2} / m_{1}$ and $k_{2}=d_{2} / m_{2}$, then (15) becomes

$$
\begin{aligned}
\dot{v}_{e}= & \left(\frac{m_{1}}{m_{2}}-1\right)\left(\xi_{2} \sin e_{\varphi}-\xi_{1} \cos e_{\varphi}\right) r_{F} \\
& -\frac{d_{2}}{m_{2}}\left(\xi_{1} \sin e_{\varphi}+\xi_{2} \cos e_{\varphi}\right) \\
& +\left(\xi_{2} \sin e_{\varphi}-\xi_{1} \cos e_{\varphi}\right) r_{L}-\dot{\xi}_{1} \sin e_{\varphi} \\
& -\dot{\xi}_{2} \cos e_{\varphi} .
\end{aligned}
$$

Define the control Lyapunov function (CLF):

$$
V_{2}=\frac{1}{2} v_{e}{ }^{2}+2 k_{3} \sin ^{2}\left(\frac{e_{\varphi}}{2}\right), \quad k_{3}>0 \in \mathbb{R} .
$$

Then we get the time derivative of (17), giving

$$
\begin{aligned}
\dot{V}_{2} & =v_{e} \dot{v}_{e}+k_{3}\left(r_{L}-r_{F}\right) \sin e_{\varphi}=v_{e}\left[\left(\frac{m_{1}}{m_{2}}-1\right) \lambda r_{F}\right. \\
& \left.-\frac{d_{2}}{m_{2}} \zeta+\lambda r_{L}-\dot{\xi}_{1} \sin e_{\varphi}-\dot{\xi}_{2} \cos e_{\varphi}\right]+k_{3} r_{L} \sin e_{\varphi} \\
& -k_{3} r_{F} \sin e_{\varphi},
\end{aligned}
$$

where $\lambda=\xi_{2} \sin e_{\varphi}-\xi_{1} \cos e_{\varphi}, \zeta=\xi_{1} \sin e_{\varphi}+\xi_{2} \cos e_{\varphi}$.

Choose the virtual control:

$$
r_{F}^{v}=r_{L}+k_{4} \sin e_{\varphi}-k_{5}\left(\frac{m_{1}}{m_{2}}-1\right) v_{e} \lambda+\Theta,
$$

where

$$
\begin{aligned}
\Theta= & -\operatorname{sgn}\left(v_{e}\right) \\
& \cdot \frac{\left[\left(k_{4}+k_{3} k_{5}\right)\left\|m_{1} / m_{2}-1\right\|+d_{2} / m_{2}\right]\|\zeta\|}{\left\|m_{1} / m_{2}-1\right\|\|\zeta\|+k_{3}} .
\end{aligned}
$$

Then (18) becomes

$$
\begin{aligned}
\dot{V}_{2} & =-k_{5}\left(\frac{m_{1}}{m_{2}}-1\right)^{2} v_{e}^{2} \lambda^{2}-k_{3} k_{4} \sin ^{2} e_{\varphi} \\
& +v_{e}\left[\lambda\left(\frac{m_{1} r_{L}}{m_{2}}+\frac{m_{1}}{m_{2}}-1\right)\left(k_{4}+k_{3} k_{5}\right) \sin e_{\varphi}\right. \\
& \left.-\dot{\xi}_{1} \sin e_{\varphi}-\dot{\xi}_{2} \cos e_{\varphi}-\frac{d_{2} \zeta}{m_{2}}\right]+\left[v_{e}\left(\frac{m_{1}}{m_{2}}-1\right) \lambda\right. \\
& \left.-k_{3} \sin e_{\varphi}\right] \Theta \leq-k_{5}\left(\frac{m_{1}}{m_{2}}-1\right)^{2} v_{e}^{2} \lambda^{2}-k_{3} k_{4} \\
& \cdot \sin ^{2} e_{\varphi}+\left\|v_{e}\right\| \\
& \cdot\left[\|\zeta\|\left(\left\|\frac{r_{L} m_{1}}{m_{2}}\right\|+\left\|\frac{m_{1}}{m_{2}}-1\right\|\left(k_{4}+k_{3} k_{5}\right)+\frac{d_{2}}{m_{2}}\right)\right. \\
& \left.+\left\|\dot{\xi}_{1}\right\|+\left\|\dot{\xi}_{2}\right\|\right]+\left(\left\|v_{e}\right\|\left\|\frac{m_{1}}{m_{2}}-1\right\|\|\zeta\|+k_{3}\right) \Theta \\
& \leq-k_{5}\left(\frac{m_{1}}{m_{2}}-1\right)^{2} v_{e}^{2} \lambda^{2}-k_{3} k_{4} \sin ^{2} e_{\varphi}+\sigma
\end{aligned}
$$

where $\sigma$ is an arbitrary bounded infinitesimal constant, so the system $\left(v_{e}, e_{\varphi}\right)$ is bounded.

3.2. Dynamics Controls. The virtual controls $u_{F}^{v}$ and $r_{F}^{v}$ are proposed; then the dynamics controls should be designed to generate torque controls $\tau_{u F}$ and $\tau_{r F}$ for the followers to make the vessels achieve the desired formation. 
Step 1. Define the velocity error in surge as $u_{e}=u_{F}-u_{F}^{v}$, and define the CLF as $V_{3}=(1 / 2) u_{e}^{2}$; then we can get $\dot{V}_{3}=$ $u_{e}\left(\left(m_{2} / m_{1}\right) v_{F} r_{F}-\left(d_{1} / m_{1}\right) u_{F}+\left(1 / m_{1}\right) \tau_{u F}-\dot{u}_{F}^{v}\right)$.

So the torque control $\tau_{u F}$ can be chosen as

$$
\tau_{u F}=m_{1}\left(-k_{6} u_{e}-\frac{m_{2}}{m_{1}} v_{F} r_{F}+\frac{d_{1}}{m_{1}} u_{F}+\dot{u}_{F}^{v}\right),
$$

$k_{6}>0 \in \mathbb{R}$.

Then $\dot{V}_{3}=-k_{6} u_{e}^{2} \leq 0$

Step 2. Define the angle velocity error as $r_{e}=r_{F}-r_{F}^{v}$, and define the CLF as $V_{4}=(1 / 2) r_{e}{ }^{2}$; then the time derivative of $V_{4}$ is given by

$$
\dot{V}_{4}=r_{e}\left(\frac{m_{1}-m_{2}}{m_{3}} u_{F} v_{F}-\frac{d_{3}}{m_{3}} r_{F}+\frac{1}{m_{3}} \tau_{r F}-\dot{r}_{F}^{v}\right) .
$$

So we can choose the torque control $\tau_{r F}$ as

$$
\begin{aligned}
& \tau_{r F}=m_{3}\left(-k_{7} r_{e}-\frac{m_{1}-m_{2}}{m_{3}} u_{F} v_{F}+\frac{d_{3}}{m_{3}} r_{F}+\dot{r}_{F}^{v}\right), \\
& k_{7}>0 \in \mathbb{R} .
\end{aligned}
$$

Then $\dot{V}_{4}=-k_{7} r_{e}^{2} \leq 0$.

Define a CLF as

$$
V_{5}=V_{1}+V_{2}+\frac{1}{2} r_{e}^{2}+\frac{1}{2} u_{e}^{2},
$$

and then the time derivative of $V_{5}$ is given by

$$
\begin{aligned}
\dot{V}_{5} & =\dot{V}_{1}+\dot{V}_{2}+r_{e} \dot{r}_{e}+u_{e} \dot{u}_{e}=\varepsilon_{1} \dot{\varepsilon}_{1}+\varepsilon_{2} \dot{\varepsilon}_{2}+v_{e} \dot{v}_{e} \\
& +k_{3}\left(r_{L}-r_{F}\right) \sin e_{\varphi}+r_{e} \dot{r}_{e}+u_{e} \dot{u}_{e}=\varepsilon_{1}\left(u_{F}\right. \\
& \left.+\xi_{1} \cos e_{\varphi}-\xi_{2} \sin e_{\varphi}\right)+\varepsilon_{2}\left(v_{F}+\xi_{1} \sin e_{\varphi}\right. \\
& \left.+\xi_{2} \cos e_{\varphi}\right)+v_{e}\left[\left(\frac{m_{1}}{m_{2}}-1\right) \lambda r_{F}-\frac{d_{2}}{m_{2}} \zeta+\lambda r_{L}\right. \\
& \left.-\dot{\xi}_{1} \sin e_{\varphi}-\dot{\xi}_{2} \cos e_{\varphi}\right]+k_{3} r_{L} \sin e_{\varphi}-k_{3} r_{F} \sin e_{\varphi} \\
& +r_{e}\left(\frac{m_{1}-m_{2}}{m_{3}} u_{F} v_{F}-\frac{d_{3}}{m_{3}} r_{F}+\frac{1}{m_{3}} \tau_{r F}-\dot{r}_{F}^{v}\right) \\
& +u_{e}\left(\frac{m_{2}}{m_{1}} v_{F} r_{F}-\frac{d_{1}}{m_{1}} u_{F}+\frac{1}{m_{1}} \tau_{u F}-\dot{u}_{F}^{v}\right) .
\end{aligned}
$$

Substituting (12), (13), (19), (22), and (24) into $\dot{V}_{5}$, we can get

$$
\begin{aligned}
\dot{V}_{5} & =-k_{1} \varepsilon_{1}{ }^{2}-k_{2} \varepsilon_{2}{ }^{2}-k_{5}\left(\frac{m_{1}}{m_{2}}-1\right)^{2} v_{e}{ }^{2} \lambda^{2}-k_{3} k_{4} \\
& \cdot \sin ^{2} e_{\varphi} \\
& +v_{e}\left[\lambda\left(\frac{m_{1} r_{L}}{m_{2}}+\frac{m_{1}}{m_{2}}-1\right)\left(k_{4}+k_{3} k_{5}\right) \sin e_{\varphi}\right. \\
& \left.-\dot{\xi}_{1} \sin e_{\varphi}-\dot{\xi}_{2} \cos e_{\varphi}-\frac{d_{2} \zeta}{m_{2}}\right]+\left[v_{e}\left(\frac{m_{1}}{m_{2}}-1\right) \lambda\right.
\end{aligned}
$$

$$
\begin{aligned}
& \left.-k_{3} \sin e_{\varphi}\right] \Theta-k_{6} u_{e}^{2}-k_{7} r_{e}^{2} \leq-k_{1} \varepsilon_{1}^{2}-k_{2} \varepsilon_{2}^{2} \\
& -k_{5}\left(\frac{m_{1}}{m_{2}}-1\right)^{2} v_{e}{ }^{2} \lambda^{2}-k_{3} k_{4} \sin ^{2} e_{\varphi}-k_{6} u_{e}{ }^{2} \\
& -k_{7} r_{e}{ }^{2}+\sigma
\end{aligned}
$$

where $\sigma$ is an arbitrary bounded infinitesimal constant, so the control system $\left(\varepsilon_{1}, \varepsilon_{2}, v_{e}, e_{\varphi}, u_{e}, r_{e}\right)$ is asymptotically stable.

3.3. Sensing Field Remapping. In this research, each USV is equipped with a vision-based sensor which has a sectorlike sensing field. In some cases leader will be out of the follower's sensing field such as turning and leader decelerating suddenly. Once this happens, followers will lose the leader's information. In particular, when the leader moves to the border of the follower's sensor, the formation will be lost easily.

There can be three ways to solve this problem. The first one is position errors remapping. When the leader is moving close to the border of the follower's sensor, we adjust the formation position errors; that is to say, we change the follower's position to ensure the sensing angle being not too close to the sensor's border. So the formation will be maintained. The second one is Heading Angle Error Remapping. When the leader is at or near the border of the follower's sensor, we adjust the follower's heading angle error; that is to say, control the follower to turn so as to ensure the sensing angle being not too close to the sensor's border. The third one is Position Errors Remapping and Heading Angle Error Remapping. This method uses the above two methods in cascade. Next how to design the three methods is shown in detail.

3.3.1. The First Method: Position Errors Remapping (PER). According to Assumption 2, we know that the position errors are measured by the on-board vision-based sensor. If the sensing angle $\alpha_{L}$ is changed, the position errors will be changed at the same time. Here we design an exponential remapping method (ERM) to adjust the sensing angle $\alpha_{L}$.

Define ERM as

$$
K_{\alpha_{L F i}^{\mathrm{Re}}}:=M_{e} \cdot\left(e^{\operatorname{sign}\left(\alpha_{L F i}\right) \cdot K_{\alpha} \cdot \alpha_{L F i}}-1\right)+N_{e},
$$

where $\alpha_{L F i}$ is the actual sensing angle of the follower $i$ relative to the leader, $M_{e}$ and $K_{\alpha}$ are the weights of ERM, and $N_{e}$ is the remapping deviation set value. So the remapping sensing angle $\alpha_{L F i}^{\mathrm{Re}}$ is defined as

$$
\alpha_{L F i}^{\mathrm{Re}}:=\alpha_{L F i} \cdot K_{\alpha_{L F i}^{\mathrm{Re}}}
$$

In this research, we assume the border of the follower's sensor is $\pm 60^{\circ}$; that is to say, the sensing constraint $2 \alpha=120^{\circ}$, $\alpha_{L F i} \in\left[-60^{\circ}, 60^{\circ}\right]$. Choose $M_{e}=1 / 3600, K_{\alpha}=1 / 8, N_{e}=$ 1 , we can make a figure using (29) to show the remapping relationship between the actual sensing angle $\alpha_{L F i}$ and the 


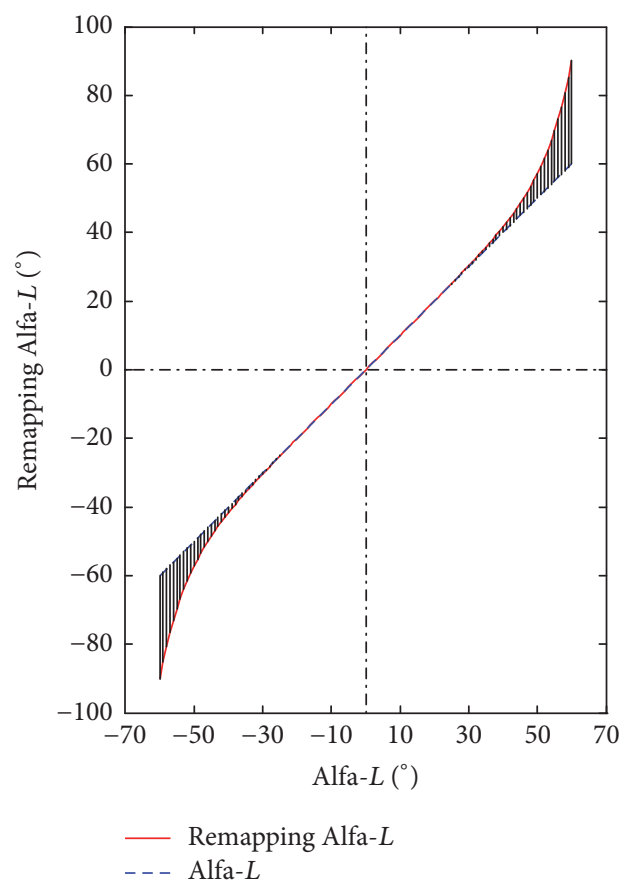

FIGURE 4: Sensing angle remapping relationship.

remapping sensing angle $\alpha_{L F i}^{\mathrm{Re}}$. Figure 4 shows the sensing angle remapping relationship.

Next $\alpha_{L F i}^{\mathrm{Re}}$ is used as the feedback sensing angle instead of the actual sensing angle, so the position errors are changed; then the follower must adjust its position to converge to zero. Figure 5 shows the position adjusting of the follower.

From Figure 5, we can get $\alpha_{L F i}=-\varphi_{F i}+\beta_{i}+\Xi$, where $\beta_{i}=\operatorname{atan}\left(\Delta Y_{i} / \Delta X_{i}\right), \beta_{i} \in(-\pi / 2,0) \cup(0, \pi / 2)$,

$$
\Xi_{i}= \begin{cases}0, & \Delta X_{i}>0 \\ \operatorname{sign}\left(\Delta Y_{i}\right) \cdot \frac{\pi}{2}+\pi, & \Delta X_{i}=0 \\ \pi, & \Delta X_{i}<0\end{cases}
$$

and $\Delta X_{i}=X_{L}-X_{F i}, \Delta Y_{i}=Y_{L}-Y_{F i}$.

The follower in Figure 5 is moving at position $F i$; the sensing angle is $\alpha_{L F i}$. However, at this time, the feedback sensing angle is $\alpha_{L F i}^{\mathrm{Re}}$, which is greater than $\alpha_{L F i}$, so the follower will change its position to make sensing angle be a smaller sensing angle $\alpha_{L F i}^{\prime}$. From Figure 5 we can see that there are many positions for the follower to achieve the sensing angle $\alpha_{L F i}^{\prime}$ as long as the follower is on the line $l_{L F i}^{\mathrm{Re}}$. For example, the sensing angle $\alpha_{L F i}^{\prime}$ will be achieved when the follower moves at positons $\widetilde{1}, \widetilde{2}, \widetilde{3}$, and $\widetilde{4}$, and so forth. However, moving to position $\widetilde{3}$ is the only shortest path, so we choose the shortest path to do the position errors remapping. Because of remapping sensing angle $\alpha_{L F i}^{\mathrm{Re}}$, we can get $\sigma_{i}=$ $\alpha_{L F i}-\alpha_{L F i}^{\mathrm{Re}}$.

The follower adjusts its position from position $F i$ to position $\widetilde{3}$, and we choose $l_{F i-\widetilde{3}}$ to denote the distance between position $F i$ and position $\widetilde{3}$. From Figure 5 we can see that

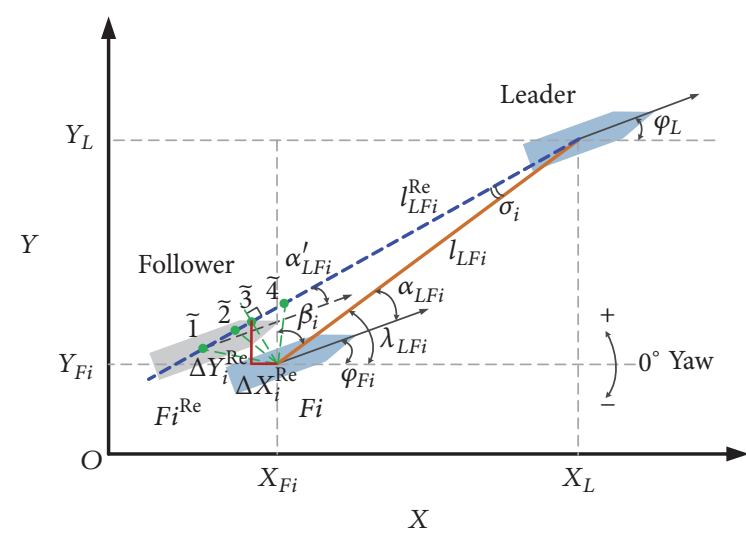

Figure 5: Follower positon adjustment.

$\Delta X_{i}^{\mathrm{Re}}$ and $\Delta Y_{i}^{\mathrm{Re}}$ are the projections of $l_{F i-\tilde{3}}$ along $X$-axis and $Y$-axis; they are calculated as follows:

$$
\begin{aligned}
\Delta X_{i}^{\mathrm{Re}} & =\sin \left(\beta_{i}-\sigma_{i}\right) \cdot l_{F i-\tilde{3}} \\
& =\sin \left(\varphi_{F i}-\Xi_{i}+\alpha_{L F i}^{\mathrm{Re}}\right) \cdot l_{F i-\tilde{3}} \\
& =\sin \left(\varphi_{F i}-\Xi_{i}+\alpha_{L F i}^{\mathrm{Re}}\right) \cdot \sin \left(\alpha_{L F i}-\alpha_{L F i}^{\mathrm{Re}}\right) \cdot l_{L F i} \\
\Delta Y_{i}^{\mathrm{Re}} & =\cos \left(\beta_{i}-\sigma_{i}\right) \cdot l_{F i-\tilde{3}} \\
& =\cos \left(\varphi_{F i}-\Xi_{i}+\alpha_{L F i}^{\mathrm{Re}}\right) \cdot l_{F i-\tilde{3}} \\
& =\cos \left(\varphi_{F i}-\Xi_{i}+\alpha_{L F i}^{\mathrm{Re}}\right) \cdot \sin \left(\alpha_{L F i}-\alpha_{L F i}^{\mathrm{Re}}\right) \cdot l_{L F i} .
\end{aligned}
$$

So the projections along $X$-axis and $Y$-axis of remapping distance $l_{L F i}^{\mathrm{Re}}$ are

$$
\begin{gathered}
\Delta X_{L-F i}^{\mathrm{Re}}=\Delta X_{L-F i}+\operatorname{sign}\left(\Delta X_{L-F i}\right) \cdot \Delta X_{i}^{\mathrm{Re}} \\
\Delta Y_{L-F i}^{\mathrm{Re}}=\Delta Y_{L-F i}-\operatorname{sign}\left(\Delta X_{L-F i}\right) \cdot \Delta Y_{i}^{\mathrm{Re}} .
\end{gathered}
$$

Using an Euler angle rotation matrix, we can get the formation remapping position as

$$
\begin{aligned}
{\left[\begin{array}{c}
l_{x i}^{\mathrm{Re}} \\
l_{y i}^{\mathrm{Re}}
\end{array}\right] } & =\left[\begin{array}{cc}
\cos \varphi_{L} & -\sin \varphi_{L} \\
\sin \varphi_{L} & \cos \varphi_{L}
\end{array}\right]\left[\begin{array}{c}
\Delta X_{L-F i}^{\mathrm{Re}} \\
\Delta Y_{L-F i}^{\mathrm{Re}}
\end{array}\right] \\
& =\left[\begin{array}{c}
\Delta X_{L-F i}^{\mathrm{Re}} \cos \varphi_{L}-\Delta Y_{L-F i}^{\mathrm{Re}} \sin \varphi_{L} \\
\Delta X_{L-F i}^{\mathrm{Re}} \sin \varphi_{L}+\Delta Y_{L-F i}^{\mathrm{Re}} \cos \varphi_{L}
\end{array}\right] .
\end{aligned}
$$

So formation error from (6) can be written as the remapping form:

$$
E^{\mathrm{Re}}=\left[\begin{array}{c}
e_{x i}^{\mathrm{Re}} \\
e_{y i}^{\mathrm{Re}}
\end{array}\right]=\left[\begin{array}{l}
l_{x i}^{\mathrm{Re}}-l_{x}^{d} \\
l_{y i}^{\mathrm{Re}}-l_{y}^{d}
\end{array}\right]
$$

Using this position remapping error as the feedback error to control the formation system will ensure that the formation is being stable and make the follower move along the shortest path to the remapping position, which will help the system get one optimal formation structure under the sensing constraints. 
3.3.2. The Second Method: Heading Angle Error Remapping (HAER). According to (7), the follower should track the yaw angle with the leader. If the leader's heading angle changes, the follower's heading angle will track the error change. So when the leader is at or near the border of the follower's sensor, we change the leader's feedback yaw angle, and the follower will turn its heading so as to ensure the sensing angle is not too close to the sensor's border. Here we design an exponential remapping method (ERM) to adjust the yaw angle $\varphi_{L}$ of the leader.

Define ERM as

$$
K_{\varphi_{L}^{\mathrm{Re}}}:= \begin{cases}0, & \left|\alpha_{L F i}\right| \leq \alpha_{L F i}^{0} \\ \operatorname{sign}\left(\alpha_{L F i}\right) \cdot\left(\exp \left(\operatorname{sign}\left(\alpha_{L F i}\right) \cdot K_{\text {yaw }} \cdot\left(\alpha_{L F i}-\operatorname{sign}\left(\alpha_{L F i}\right) \cdot \alpha_{L F i}^{0}\right)\right)-1\right), & \alpha_{L F i}^{0} \leq\left|\alpha_{L F i}\right| \leq \alpha_{L F i}^{\max },\end{cases}
$$

where $\alpha_{L F i}$ is the actual sensing angle of the follower $i$ relative to the leader, $K_{\text {yaw }}$ is the weight of ERM, $\alpha_{L F i}^{0}$ is the given safe sensing angle, abd $\alpha_{L F i}^{\max }$ is max sensing angle. So the remapping heading angle is defined as

$$
\varphi_{L}^{\mathrm{Re}}:=\varphi_{L}+K_{\varphi_{L}^{\mathrm{Re}}}
$$

So heading angle error from (7) can be wrote as the remapping form:

$$
e_{\varphi}=\varphi_{L}^{\mathrm{Re}}-\varphi_{F}
$$

Using this heading angle remapping error as the feedback error to control the formation system will keep the follower being sensing with the leader.

In this research, we assume the border of the follower's sensor is $\pm 60^{\circ}$; that is to say, the sensing constraint $2 \alpha=120^{\circ}$, $\alpha_{L F i} \in\left[-60^{\circ}, 60^{\circ}\right], \alpha_{L F i}^{\max }=60^{\circ}$. Choosing $K_{\text {yaw }}=0.185, \alpha_{L F i}^{0}=$ $45^{\circ}$, we can make a figure (Figure 6) using (36) to show the remapping relationship between the actual sensing angle $\alpha_{L F i}$ and the remapping value $K_{\varphi_{L}^{\mathrm{Re}}}$.

3.3.3. The Third Method: Position Errors Remapping and Heading Angle Error Remapping (PER\&HAER). This method uses the above two methods in cascade.

\section{Simulation and Discussion}

To demonstrate the effectiveness of the control scheme proposed for leader-follower formation control of USVs with vision-based sensing constraints, several computer simulations are performed. Three USVs with the same parameters are chosen and modeled. The vehicles' parameters are

$$
\begin{aligned}
& m_{1}=120 \times 10^{3} \mathrm{~kg}, \\
& m_{2}=217.9 \times 10^{3} \mathrm{~kg}, \\
& m_{3}=636 \times 10^{5} \mathrm{~kg}, \\
& d_{1}=215 \times 10^{2} \mathrm{~kg} \cdot \mathrm{s}^{-1}, \\
& d_{2}=117 \times 10^{3} \mathrm{~kg} \cdot \mathrm{s}^{-1}, \\
& d_{3}=802 \times 10^{4} \mathrm{~kg} \cdot \mathrm{s}^{-1},
\end{aligned}
$$

which are taken from [37]. First, three methods proposed in Section 3 are simulated, respectively. Second, we make comparisons and analysis of the three methods. Last, a triangular formation of three USVs is simulated using the best one of the three methods.

4.1. Several Cases Results. Three cases of simulation are done in this section. The border of the follower's sensor is set as $\pm 60^{\circ}$, that is to say the sensing constraint $2 \alpha=120^{\circ}$, sensing angle $\alpha_{L F i} \in\left[-60^{\circ}, 60^{\circ}\right]$, and the max sensing angle $\alpha_{L F i}^{\max }=60^{\circ}$. We choose two USV to do the simulations; one of them is used as the leader, and another one is the follower. The desired formation is chosen as $\mathbb{F}_{L F 1}^{d}=\left[\begin{array}{ll}l_{L F 1}^{d} & \lambda_{L F 1}^{d}\end{array}\right]^{T}=$ $[20,2 \pi / 3]^{T}$. It is easy to know that the sensing angle $\alpha_{L F 1}$ is expected to converge to $-60^{\circ}$ when $\lambda_{L F 1}^{d}$ is chosen as $2 \pi / 3$. However the sensing field is limited between $-60^{\circ}$ and $60^{\circ}$; this means the leader is at the border of the follower's sensing field, which is dangerous for the formation. So we use this desired formation to demonstrate the proposed control strategies. Other parameters of PER and HAER are chosen according to Section 3.3.

Case 1 (position errors remapping (PER)). In this case, the initial positions of the vehicles are chosen as Leader: $\left[X_{L}, Y_{L}\right]=[0,0]$, Follower: $\left[X_{F 1}, Y_{F 1}\right]=[-40,50]$. And the initial velocities are chosen as Leader: $u_{L}=1 \mathrm{~m} / \mathrm{s}, v_{L}=0 \mathrm{~m} / \mathrm{s}$, $r_{L}=0 \mathrm{rad} / s ;$ Follower: $\left[u_{F 1}, v_{F 1}, r_{F 1}\right]=[0,0,0]$.

The simulation results are shown in Figures 7 and 8 . The formation trajectories of the two USV using PER are shown in Figure 7, and Figure 8 shows the sensing angle of follower 1 relative to the leader using PER. The total simulation time is 1000 seconds. The leader's yaw angle velocity and the surge velocity are assumed to remain unchanged during the simulation. $\alpha_{L F i}^{\mathrm{Re}}$ from (28) is set to be 0 before 300 th second, which means before 300th PER does not work. After 300th second, the PER begins to work.

From the simulation results, we can see that, before the PER works, the formation converges to be stable, and the sensing angle is stabilized at $-60^{\circ}$, which is not safe for keeping the formation. At 300th second, the PER begins to work; under the control of PER, the formation shrinks; however, the sensing angle converges to be around $-48^{\circ}$, which will make the formation be more safe and stable. 


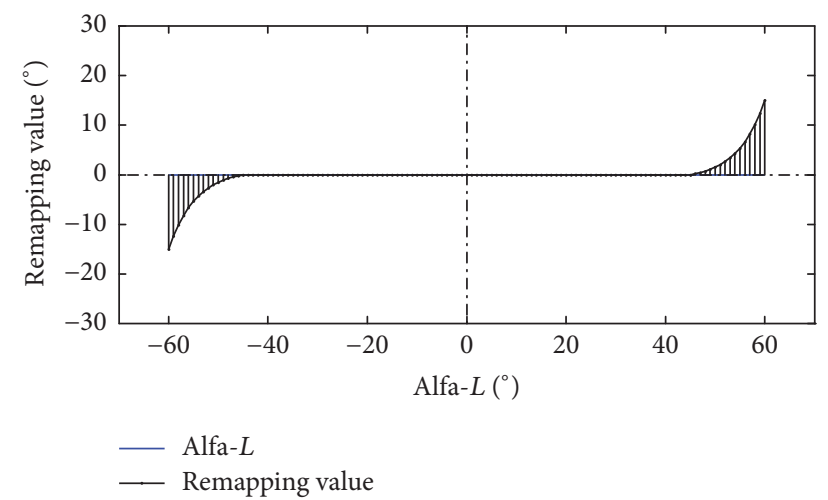

FIGURE 6: Relationship between actual sensing angle and remapping value.

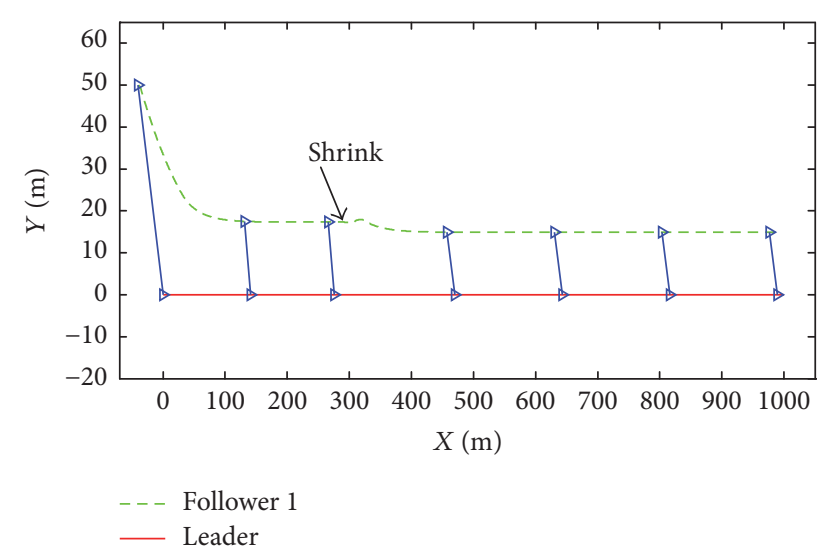

FIGURE 7: Formation trajectories using PER.

Case 2 (heading angle error remapping (HAER)). In this case, the initial positions of the vehicles are chosen as follows:

Leader: $\left[X_{L}, Y_{L}\right]=[0,0]$, Follower: $\left[X_{F 1}, Y_{F 1}\right]=$ $[-40,50]$

The initial velocities are as follows:

$$
\begin{aligned}
& \text { Leader: } u_{L}=1 \mathrm{~m} / \mathrm{s}, v_{L}=0 \mathrm{~m} / \mathrm{s}, r_{L}=0 \mathrm{rad} / \mathrm{s} \\
& \text { Follower: }\left[u_{F 1}, v_{F 1}, r_{F 1}\right]=[0,0,0]
\end{aligned}
$$

The given safe sensing angle $\alpha_{L F 1}^{0}$ is $45^{\circ}$.

The simulation results are shown in Figures 9 and 10 . The formation trajectories of the two USVs using HAER are shown in Figure 9, and Figure 10 shows the sensing angle of follower 1 relative to the leader using HAER. The total simulation time is 1000 seconds. The leader's yaw angle velocity and the surge velocity are assumed to remain unchanged during the simulation. $K_{\varphi_{L}^{\mathrm{Re}}}$ from (35) is set to be 0 before 300th second, which means before 300th HAER does not work. After 300th second, the HAER begins to work.

From the simulation results, we can see that, before the HAER works, the formation converges to be stable, and the sensing angle is stabilized at $-60^{\circ}$, which is not safe for keeping the formation. At 300th second, the HAER begins

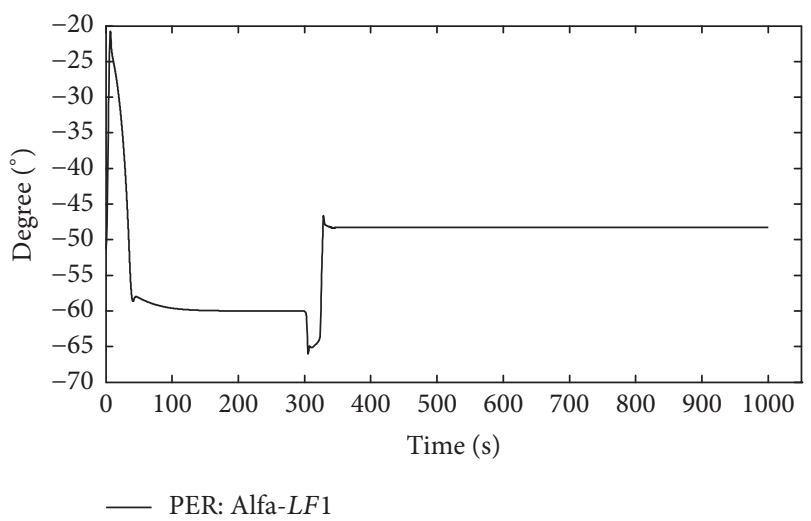

FIGURE 8: Sensing angle of follower 1 relative to the leader using PER.

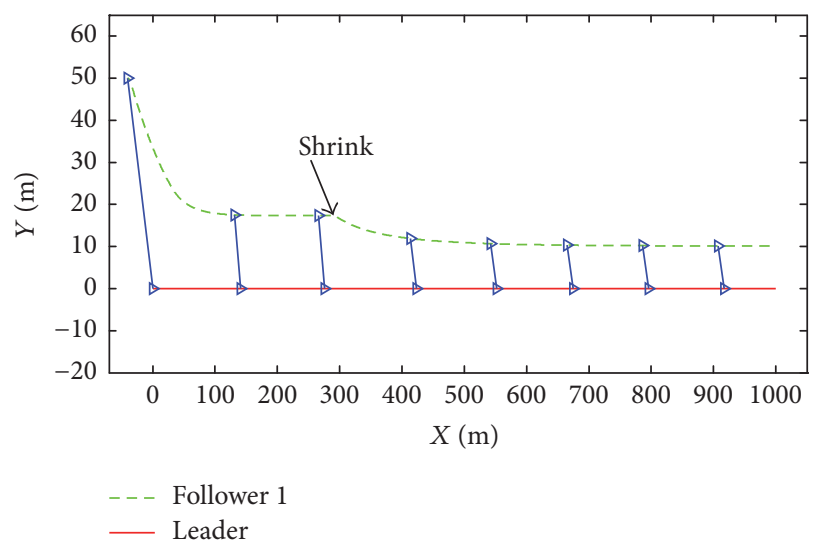

FIgURE 9: Formation trajectories using HAER.

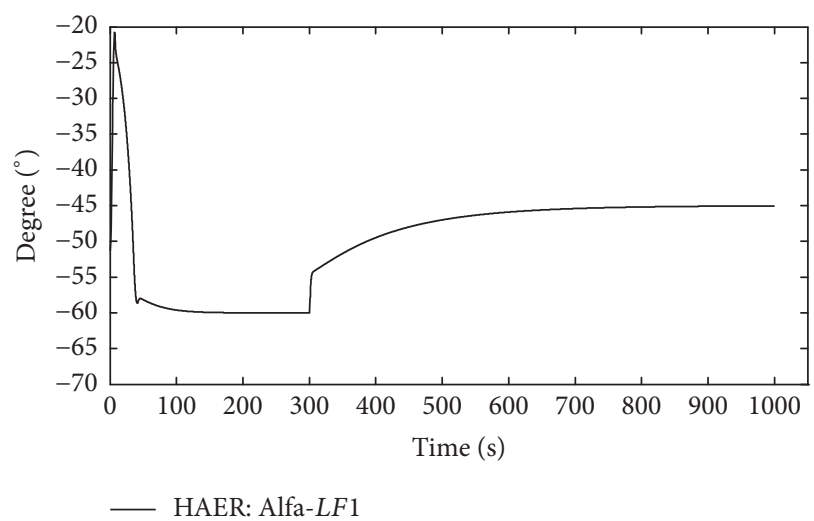

FIGURE 10: Sensing angle of follower 1 relative to the leader using HAER.

to work; under the control of HAER, the formation shrinks; however the sensing angle converges to the desired safe sensing angle $-45^{\circ}$, which will ensure that the follower keeps sensing with the leader.

Case 3 (double remapping (PER\&HAER)). This case uses the above two methods PER and HAER in cascade. All of the parameters are the same as those in Cases 1 and 2. 


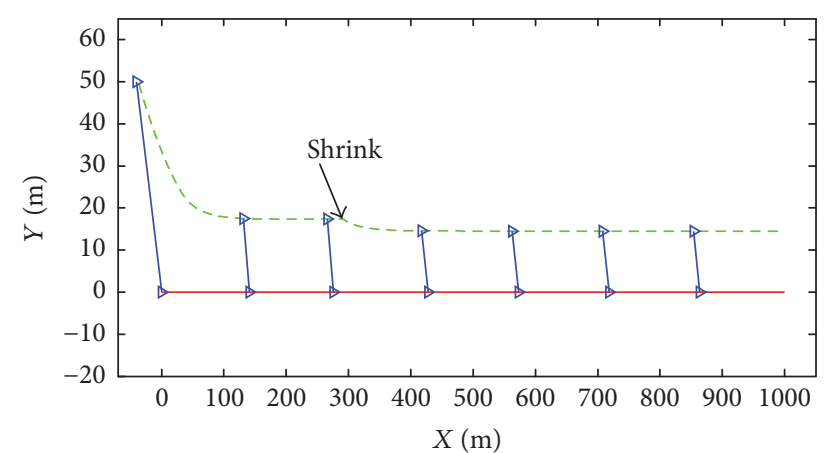

- Follower 1

— Leader

FIgURE 11: Formation trajectories using PER\&HAER.

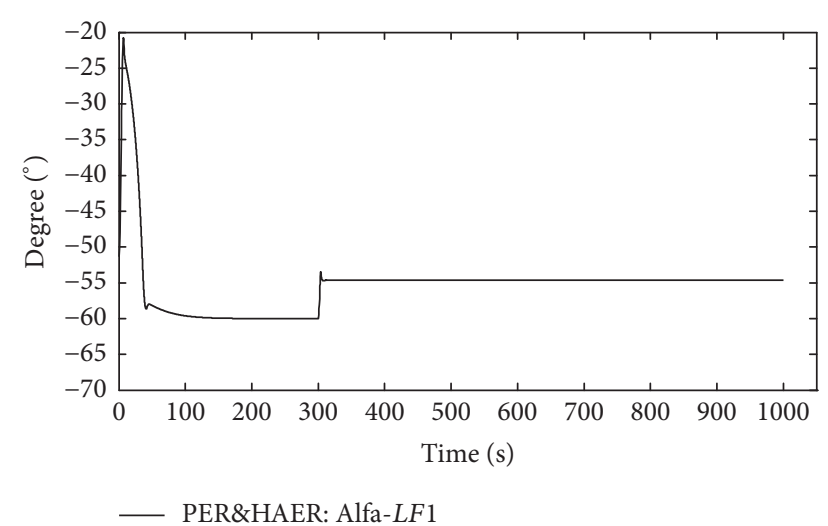

FIGURE 12: Sensing angle of follower 1 relative to the leader using PER\&HAER.

The simulation results are shown in Figures 11 and 12. The formation trajectories of the two USVs using double remapping PER\&HAER are shown in Figure 11, and Figure 12 shows the sensing angle of follower 1 relative to the leader. The total simulation time is 1000 seconds. $\alpha_{L F i}^{\mathrm{Re}}$ from (28) and $K_{\varphi_{L}^{\mathrm{Re}}}$ from (35) are set to be 0 before 300th second, which means before 300th PER\&HAER does not work. After 300th second, the double remapping begins to work.

From the simulation results, we can see that the formation shrinks after PER\&HAER works, and the sensing angle converges to be around $-55^{\circ}$, which will ensure that the follower keeps sensing with the leader.

4.2. Comparison and Analysis. The differences between the above three cases are shown in this section. Next we will make some comparisons in three ways to analyze the characteristics and effectiveness of the three different methods.

4.2.1. Convergence Rate. Figure 13 shows the comparison of formation trajectories. All the four formation trajectories are able to converge to be stable. However, after 300th second, the ERM begins to work, the formation convergence rate is the slowest using HAER. The formation convergence rates using PER and PER\&HAER are close. But there exists overshoot

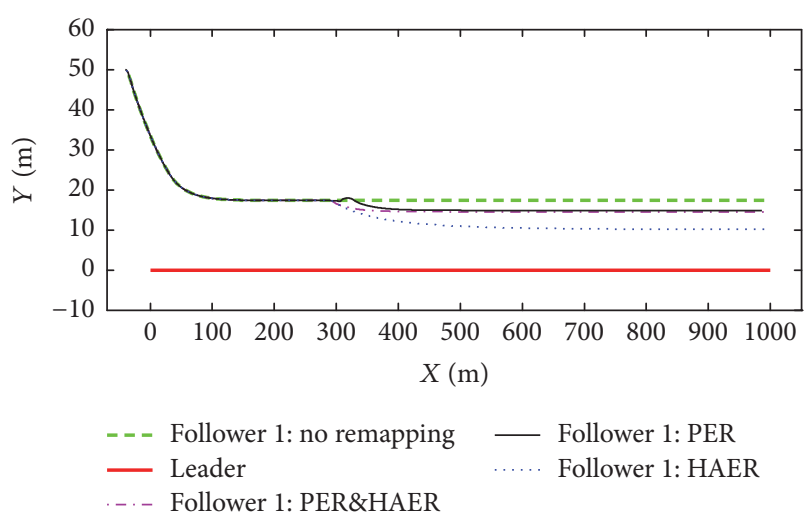

FIGURE 13: Comparison of formation trajectories.

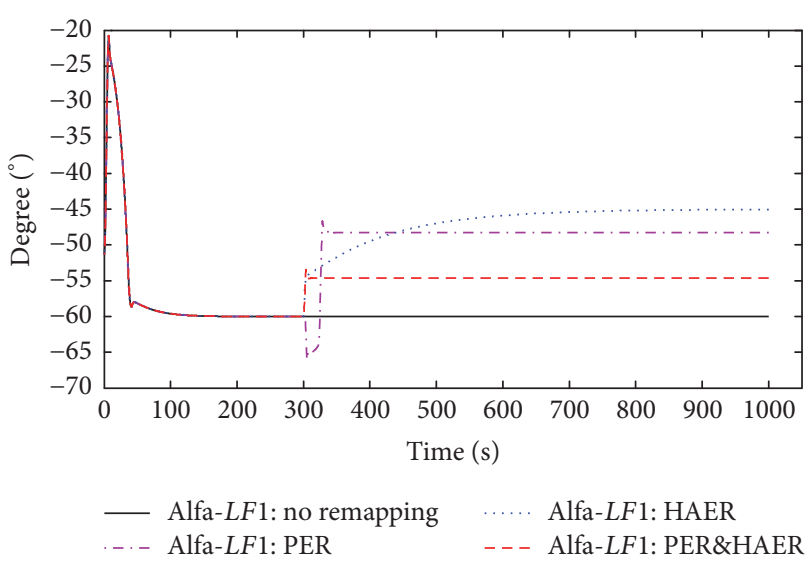

FIGURE 14: Comparison of sensing angles.

using PER. Compared with "no remapping" formation trajectory, the formation trajectory using HAER shrinks more than the formation trajectories using PER\&HAER and PER, which means its formation is worst. There is little difference between PER\&HAER trajectory and PER trajectory, and the two methods are better than HAER.

4.2.2. Safety. The simulation results of sensing angle shown together in Figure 14. From Figure 13 we know that all the four formation trajectories converge to be stable. So the sensing angles are also stable in the end. In this research, the border of the follower's sensor is $\pm 60^{\circ}$. Figure 14 shows that under no remapping the sensing angle is stabilized at $-60^{\circ}$, and this is dangerous for the formation. And the sensing angles converge to be around $-45^{\circ},-48^{\circ}$, and $-54^{\circ}$ using HAER, PER, and PER\&HAER, respectively, whose absolute values are smaller than the sensing border angle, so they can ensure that the follower keeps sensing with the leader. We can see that all the three remapping methods can be able to adjust the sensing angle. The sensing angle is safest using HAER method, but it has a slow convergence. The other two methods can converge quickly. However the sensing angle has negative overshoot using PER and, during 300th second and 320 th second the sensing angle, is beyond $-60^{\circ}$, which means 


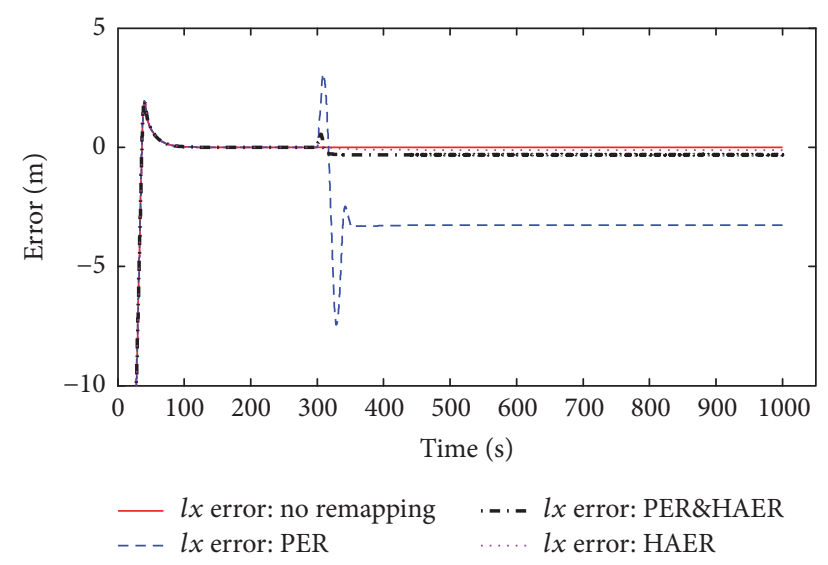

FIGURE 15: Comparison of formation errors in $l_{x}$ direction.

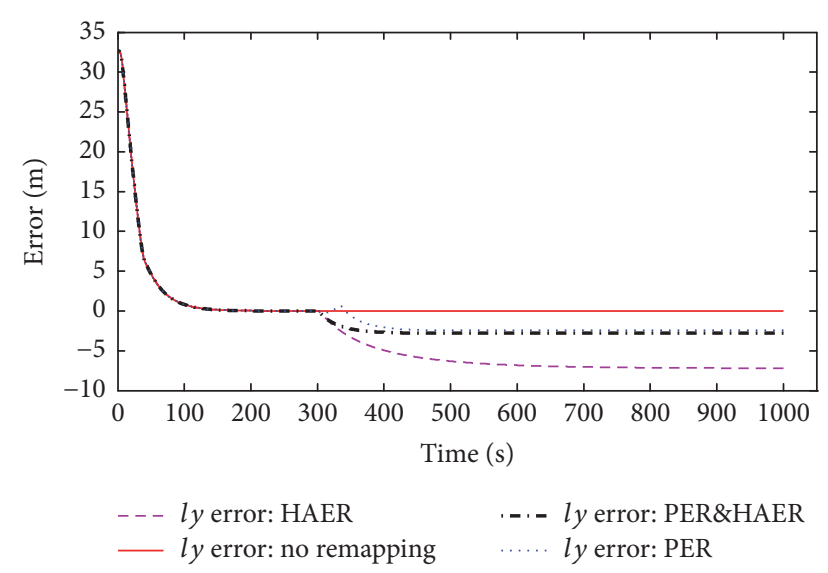

FIGURE 16: Comparison of formation errors in $l_{y}$ direction.

the worst situation happens: vision lost. Thus PER\&HAER achieves the best result.

4.2.3. Formation Errors. The core idea of ERM is to design a remapping error as the feedback for the control. It controls the followers to change their positions to converge to the remapping errors rather than the actual errors. So there must be actual steady-state errors. Figures 15 and 16 show the formation errors in $l_{x}$ direction and $l_{y}$ direction. The steady formation error in $l_{y}$ direction using PER is smallest compared with the errors using HAER and PER\&HAER; however, it achieves the biggest error in $l_{x}$ direction. The steady formation error in $l_{x}$ direction using HAER achieves the smallest value compared with the errors using PER and PER\&HAER; however, it is biggest in $l_{y}$ direction. Compared with the errors in $l_{x}$ direction and $l_{y}$ direction using PER and HAER, the PER\&HAER method achieves the best formation error. However there is also yaw angle steady-state error using PER\&HAER, which is shown in Figure 17.

According to the above comparisons from Figures 13-17, we can see all the three methods can be able to deal with the sensing constraints problem; however there exist different effects. The HAER method can be able to achieve a safest

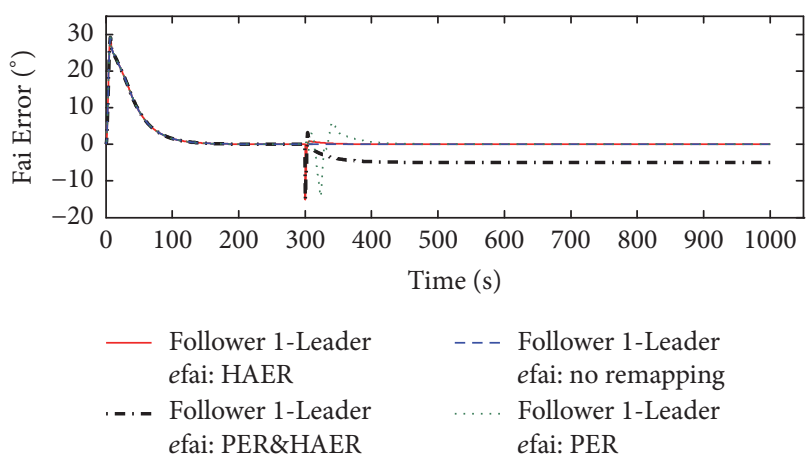

FIGURE 17: Comparison of yaw tracking errors.

formation ensuring the follower keeping sensing with the leader; however its convergence speed is slow, and the steadystate formation error in $l_{y}$ direction is biggest. The advantage of PER is that it can be able to make the follower reach its remapping position along an optimal path; however its remapping sensing angle has undershoot and the steady-state formation error in $l_{x}$ direction is biggest. The PER\&HAER method owns the advantages of the other two methods, so PER\&HAER is the best one.

4.3. Three USVs Simulation Results. According to the proposed ERM, the intensity of remapping result is decided by the EPM parameters. The more intense the remapping is, the safer the formation will be, but the more errors the formation will achieve. Though all the three methods may achieve the best formation, PER and HAER have their own disadvantages, which are undershoot and slow convergence. Considering the comparisons from the three methods, PER\&HAER method which is better than the other two methods is chosen. A triangular formation of three USVs is simulated here using PER\&HAER. The initial positions of the vehicles are chosen as Leader: $\left[X_{L}, Y_{L}\right]=[0,0]$, Follower $1:\left[X_{F 1}, Y_{F 1}\right]=$ $[-40,50]$, Follower 2: $\left[X_{F 2}, Y_{F 2}\right]=[-40,-50]$. The initial velocities are as follows.

Leader: $u_{L}=1 \mathrm{~m} / \mathrm{s}, v_{L}=0 \mathrm{~m} / \mathrm{s}, r_{L}=0 \mathrm{rad} / \mathrm{s}$ (during the simulation time $0 \sim 200 \mathrm{~s}$ ), $r_{L}=0.01 \mathrm{rad} / \mathrm{s}$ (during the simulation time 200 260s), $r_{L}=-0.01 \mathrm{rad} / \mathrm{s}$ (during the simulation time 260 410 s), $r_{L}=0.01 \mathrm{rad} / \mathrm{s}$ (during the simulation time $410 \sim 550 \mathrm{~s}$ ), $r_{L}=0 \mathrm{rad} / \mathrm{s}$ (during the simulation time 550 1000 s).

Follower 1: $\left[u_{F 1}, v_{F 1}, r_{F 1}\right]=[0,0,0]$, Follower 2: $\left[u_{F 2}, v_{F 2}\right.$, $\left.r_{F 2}\right]=[0,0,0]$. The other parameters are set to be the same as in Section 4.1. The total simulation time is 1000 seconds; the leader's initial angle velocity in yaw is set to be $0 \mathrm{rad} / \mathrm{s}$ before 200 th second, and changes to $0.01 \mathrm{rad} / \mathrm{s}$ at 200th second, then turns to $-0.01 \mathrm{rad} / \mathrm{s}$ at 260 th second, turns to be $0.01 \mathrm{rad} / \mathrm{s}$ again at 410th second, and keeps $0 \mathrm{rad} / \mathrm{s}$ during 550th to the end. The surge velocity of the leader is assumed to remain unchanged during the simulation.

Two simulations were done: one is the triangular formation control simulation which did not use the PER\&HAER method and another one used PER\&HAER. Figure 18 shows the triangular formation trajectories using no remapping 


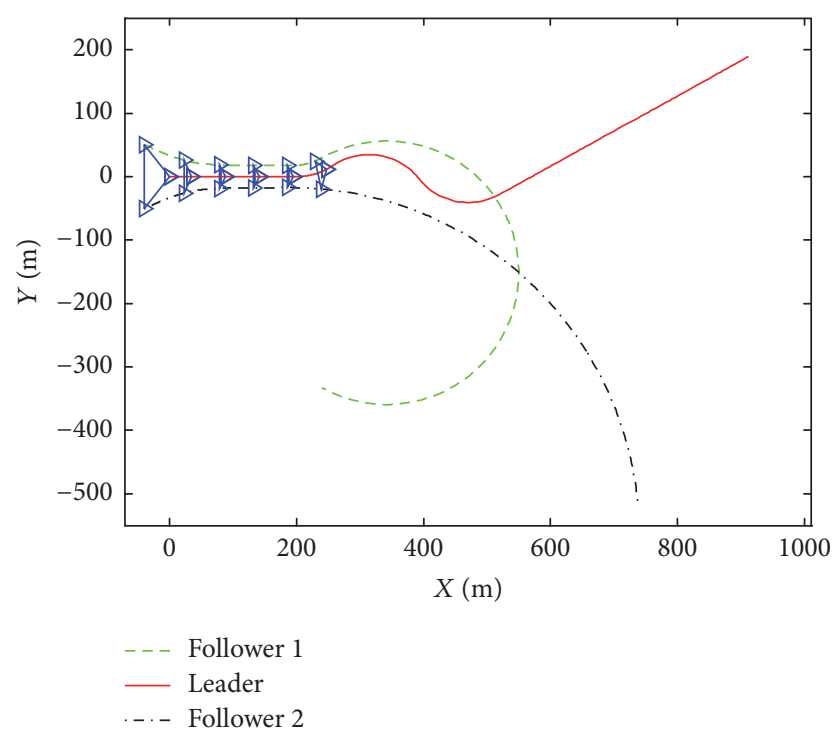

FIGURE 18: Triangular formation trajectories using no remapping method.

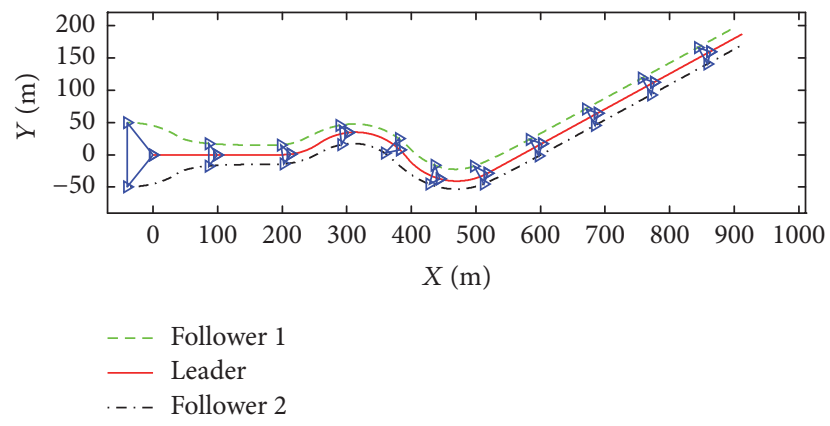

FIGURE 19: Triangular formation trajectories using PER\&HAER.

method. We can see that the formation collapses after around 230th second. This is because of the leader's turning which results in the fact that it moves out of the follower's sensing field and losing the leader's information.

Figure 19 shows the triangular formation trajectories using PER\&HAER; the formation converges to be stable. And the sensing angles of the two followers relative to the leader using PER\&HAER are shown in Figure 20. We can see that in Figure 20(a) follower 1's sensing angle converges to be around $-48.5^{\circ}$, and in Figure 20(b) follower 2's sensing angle is around $48.5^{\circ}$, all of which are not beyond the sensing constraint angle.

As one can see, the USVs achieve the stable formation successfully for both straight-line and curved-line paths. And the followers keep sensing with the leader effectively. This means that PER\&HAER works well. Triangular formation errors using PER\&HAER are shown in Figure 21, which can be able to converge to be stable. Therefore, from the simulation results, one can see that the proposed control system is effective in achieving the stable formation dealing with sensing constraints.

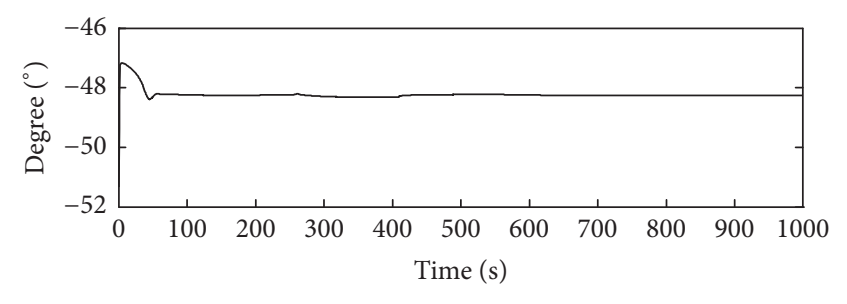

— Alfa- $L F 1:$ PER\&HAER

(a)

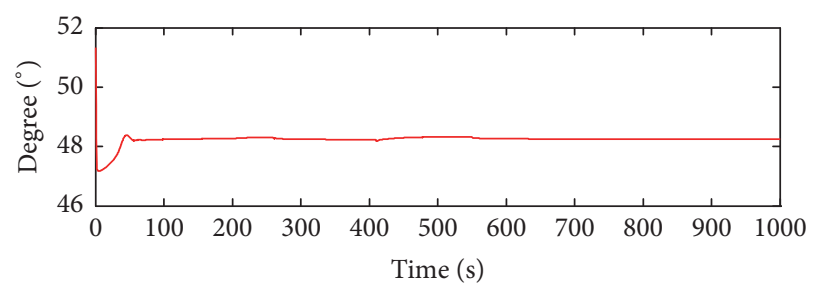

— Alfa- $L F 2$ : PER\&HAER

(b)

FIGURE 20: Sensing angles of two followers relative to the leader using PER\&HAER.

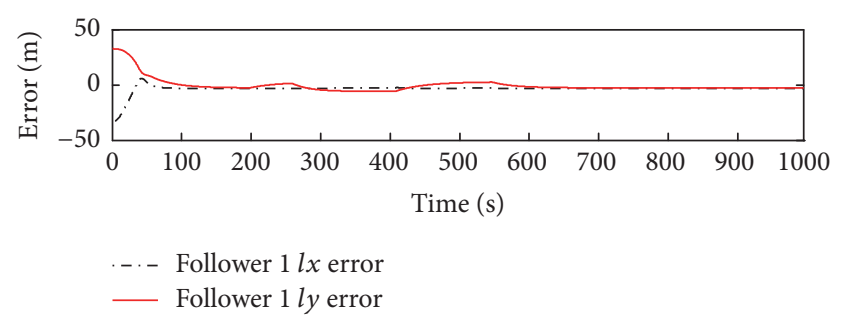

(a)

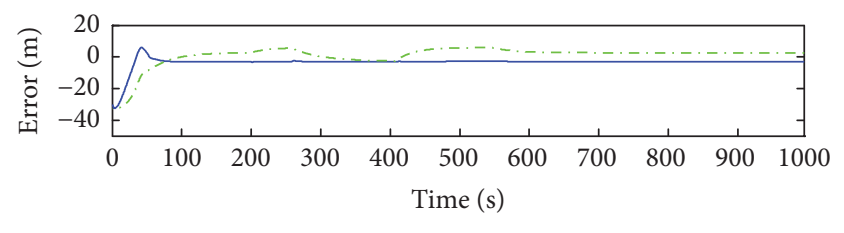

- Follower $2 l x$ error

-..- Follower 2 ly error

(b)

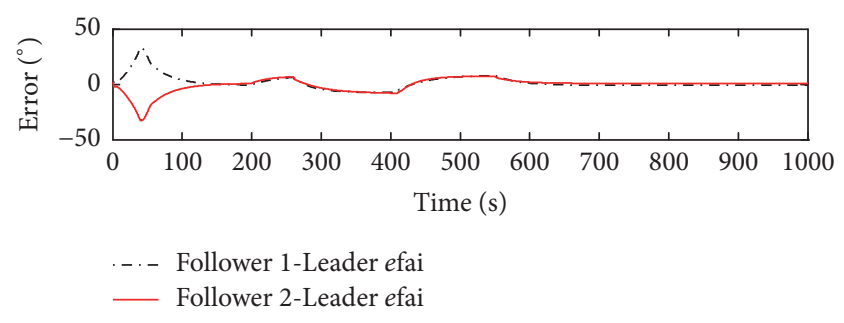

(c)

FIGURE 21: Triangular formation errors using PER\&HAER.

\section{Conclusion}

This paper presents a formation control strategy for USVs under sensing constraints. The controller uses backstepping 
method which makes it possible to achieve the desired formation under the leader-follower strategy. Three exponential remapping methods dealing with the sensing constraints problem are proposed to track straight-line as well as curvedline paths, which can be able to make the followers keep sensing with the leader so as to maintain the stable formation. Comparisons are done among the three methods. Simulation results demonstrate the effectiveness of the proposed control scheme PER\&HAER for achieving a stable triangular formation tracking straight-line and curved-line paths. This control method does not account for environmental disturbances, and the sensor error problems such as noise, random error, and sampling rate which are inherent in the sensor are not taken in account either. For real applications, this should be considered in further researches.

\section{Conflicts of Interest}

The authors declare that there are no conflicts of interest regarding the publication of this paper.

\section{References}

[1] Z. Peng, D. Wang, Z. Chen, X. Hu, and W. Lan, "Adaptive dynamic surface control for formations of autonomous surface vehicles with uncertain dynamics," IEEE Transactions on Control Systems Technology, vol. 21, no. 2, pp. 513-520, 2013.

[2] A. K. Das, R. Fierro, V. Kumar, J. P. Ostrowski, J. Spletzer, and C. J. Taylor, "A vision-based formation control framework," IEEE Transactions on Robotics and Automation, vol. 18, no. 5, pp. 813825, 2002.

[3] L. Consolini, F. Morbidi, D. Prattichizzo, and M. Tosques, "Leader-follower formation control of nonholonomic mobile robots with input constraints," Automatica. A Journal of IFAC, the International Federation of Automatic Control, vol. 44, no. 5, pp. 1343-1349, 2008.

[4] R. Cui, S. S. Ge, B. Voon Ee How, and Y. Sang Choo, "Leaderfollower formation control of underactuated autonomous underwater vehicles," Ocean Engineering, vol. 37, no. 17-18, pp. 1491-1502, 2010.

[5] G. L. Mariottini, F. Morbidi, D. Prattichizzo et al., "Visionbased localization for leader-Follower formation control," IEEE Transactions on Robotics, vol. 25, no. 6, pp. 1431-1438, 2009.

[6] R. W. Beard, J. Lawton, and F. Y. Hadaegh, "A coordination architecture for spacecraft formation control," IEEE Transactions on Control Systems Technology, vol. 9, no. 6, pp. 777-790, 2001.

[7] H. Mehrjerdi, J. Ghommam, and M. Saad, "Nonlinear coordination control for a group of mobile robots using a virtual structure," Mechatronics, vol. 21, no. 7, pp. 1147-1155, 2011.

[8] T. Balch and R. C. Arkin, "Behavior-based formation control for multirobot teams," IEEE Transactions on Robotics and Automation, vol. 14, no. 6, pp. 926-939, 1998.

[9] F. Arrichiello, S. Chiaverini, and T. I. Fossen, "Formation control of underactuated surface vessels using the null-spaeebased behavioral control," in Proceedings of the 2006 IEEE/RSJ International Conference on Intelligent Robots and Systems, IROS 2006, pp. 5942-5947, chn, October 2006.
[10] J. A. Fax and R. M. Murray, "Information flow and cooperative control of vehicle formations," Institute of Electrical and Electronics Engineers. Transactions on Automatic Control, vol. 49, no. 9, pp. 1465-1476, 2004.

[11] X. Cai and M. D. Queiroz, "Adaptive rigidity-based formation control for multirobotic vehicles with dynamics," IEEE Transactions on Control Systems Technology, vol. 23, no. 1, pp. 389-396, 2015.

[12] W. Dong and J. A. Farrell, "Formation control of multiple underactuated surface vessels," IET Control Theory Applications, vol. 2, no. 12, pp. 1077-1085, 2008.

[13] V. Gazi and R. Ordóñez, "Target tracking using artificial potentials and sliding mode control," International Journal of Control, vol. 80, no. 10, pp. 1626-1635, 2007.

[14] I.-A. F. Ihle, J. Jouffroy, and T. I. Fossen, "Formation control of marine surface craft: a lagrangian approach," IEEE Journal of Oceanic Engineering, vol. 31, no. 4, pp. 922-934, 2006.

[15] M. Breivik, V. E. Hovstein, and T. I. Fossen, "Ship formation control: A guided leader-follower approach," in IFAC Proceedings Volumes (IFAC-PapersOnline), vol. 17, pp. 16008-16014, 1 edition, 2008.

[16] M. Burger, A. Pavlov, E. Borhaug, and K. Y. Pettersen, "Straight line path following for formations of underactuated surface vessels under influence of constant ocean currents," in Proceedings of the 2009 American Control Conference, ACC 2009, pp. 30653070, 2009.

[17] E. Kyrkjebø, K. Y. Pettersen, M. Wondergem, and H. Nijmeijer, "Output synchronization control of ship replenishment operations: theory and experiments," Control Engineering Practice, vol. 15, no. 6, pp. 741-755, 2007.

[18] J. Ghommam and M. Saad, "Backstepping-based cooperative and adaptive tracking control design for a group of underactuated AUVs in horizontal plan," International Journal of Control, vol. 87, no. 5, pp. 1076-1093, 2014.

[19] B. S. Park, "Adaptive formation control of underactuated autonomous underwater vehicles," Ocean Engineering, vol. 96, pp. 1-7, 2015.

[20] E. Borhaug, A. Pavlov, R. Ghabcheloo, K. Pettersen, A. Pascoal, and C. Silvestre, "Formation control of underactuated marine vehicles with communication constraints," in Proceedings of the in Proceedings 7th IFAC Conference on Manoeuvring and Control of Marine Craft, 2006.

[21] R. Ghabcheloo, A. P. Aguiar, A. Pascoal, C. Silvestre, I. Kaminer, and J. Hespanha, "Coordinated path-following in the presence of communication losses and time delays," SIAM Journal on Control and Optimization, vol. 48, no. 1, pp. 234-265, 2009.

[22] J. P. Desai, J. P. Ostrowski, and V. Kumar, "Modeling and control of formations of nonholonomic mobile robots," IEEE Transactions on Robotics and Automation, vol. 17, no. 6, pp. 905908, 2001.

[23] K. D. Do, "Bounded controllers for formation stabilization of mobile agents with limited sensing ranges," Institute of Electrical and Electronics Engineers. Transactions on Automatic Control, vol. 52, no. 3, pp. 569-576, 2007.

[24] A. Ganguli, J. Cortés, and F. Bullo, "Multirobot rendezvous with visibility sensors in nonconvex environments," IEEE Transactions on Robotics, vol. 25, no. 2, pp. 340-352, 2009.

[25] K. D. Do, "Practical formation control of multiple underactuated ships with limited sensing ranges," Robotics and Autonomous Systems, vol. 59, no. 6, pp. 457-471, 2011. 
[26] D. Panagou and K. J. Kyriakopoulos, "Cooperative formation control of underactuated marine vehicles for target surveillance under sensing and communication constraints," in Proceedings of the 2013 IEEE International Conference on Robotics and Automation, ICRA 2013, pp. 1871-1876, deu, May 2013.

[27] P. Salaris, D. Fontanelli, L. Pallottino, and A. Bicchi, "Shortest paths for a robot with nonholonomic and field-of-view constraints," IEEE Transactions on Robotics, vol. 26, no. 2, pp. 269281, 2010.

[28] S. Maniatopoulos, D. Panagou, and K. J. Kyriakopoulos, "Model predictive control for the navigation of a nonholonomic vehicle with field-of-view constraints," in Proceedings of the 2013 1st American Control Conference, ACC 2013, pp. 3967-3972, 2013.

[29] J. Woo and N. Kim, "Vision-based target motion analysis and collision avoidance of unmanned surface vehicles," Proceedings of the Institution of Mechanical Engineers Part M: Journal of Engineering for the Maritime Environment, vol. 230, no. 4, pp. 566-578, 2016.

[30] H. Wang, X. Mou, W. Mou et al., "Vision based long range object detection and tracking for unmanned surface vehicle," in Proceedings of the 7th IEEE International Conference on Cybernetics and Intelligent Systems, CIS 2015 and the 7th IEEE International Conference on Robotics, Automation and Mechatronics, RAM 2015, pp. 101-105, khm, July 2015.

[31] A. J. Sinisterra, M. R. Dhanak, and K. Von Ellenrieder, "Stereo vision-based target tracking system for an USV," in Proceedings of the 2014 Oceans-St. John's, OCEANS 2014, pp. 1-7, 2014.

[32] Y. Cho, J. Park, M. Kang, and J. Kim, "Autonomous detection and tracking of a surface ship using onboard monocular vision," in Proceedings of the 12th International Conference on Ubiquitous Robots and Ambient Intelligence, URAI 2015, pp. 26-31, kor, October 2015.

[33] J. Wang, J.-Y. Liu, and H. Yi, "Formation control of unmanned surface vehicles with vision sensor constraints," in Proceedings of the MTS/IEEE Washington, OCEANS 2015, Wash, USA, October 2015.

[34] D. Chwa, "Global tracking control of underactuated ships with input and velocity constraints using dynamic surface control method," IEEE Transactions on Control Systems Technology, vol. 19, no. 6, pp. 1357-1370, 2011.

[35] T. I. Fossen, Guidance and Control of Ocean Vehicles, John Wiley and Sons Inc., 1994.

[36] X. Li, J. Xiao, and Z. Cai, "Backstepping based multiple mobile robots formation control," in Proceedings of the IEEE IRS/RSJ International Conference on Intelligent Robots and Systems, IROS 2005, pp. 1313-1318, can, August 2005.

[37] K. D. Do, Z. P. Jiang, and J. Pan, "Underactuated ship global tracking under relaxed conditions," Institute of Electrical and Electronics Engineers. Transactions on Automatic Control, vol. 47, no. 9, pp. 1529-1536, 2002. 


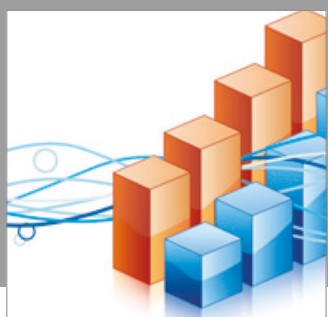

Advances in

Operations Research

vatersals

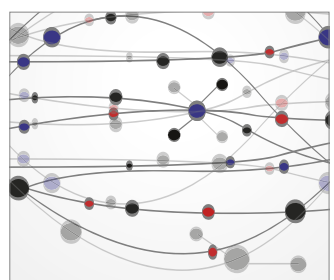

\section{The Scientific} World Journal
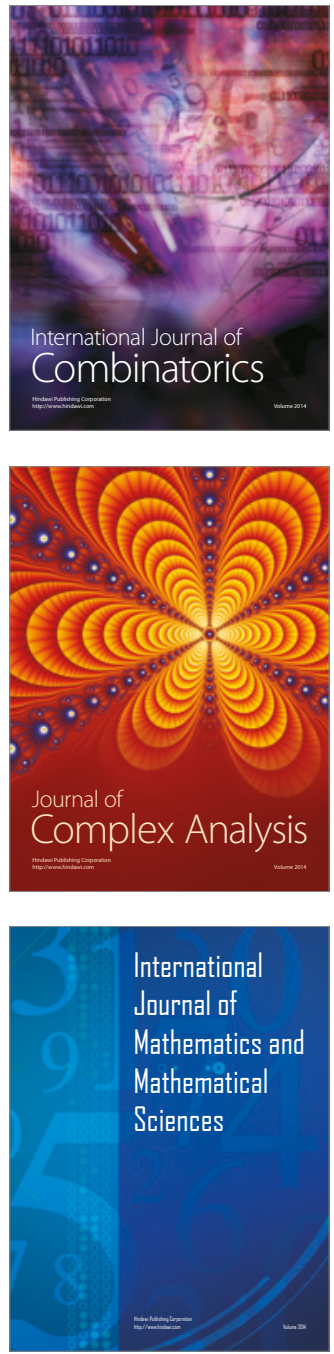
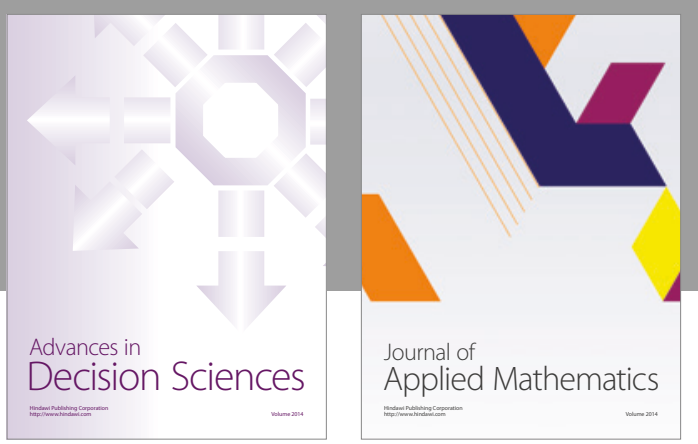

Algebra

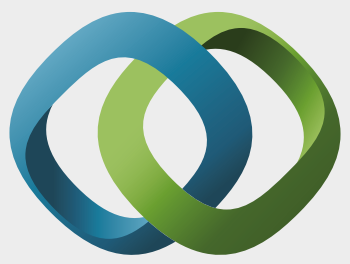

\section{Hindawi}

Submit your manuscripts at

https://www.hindawi.com
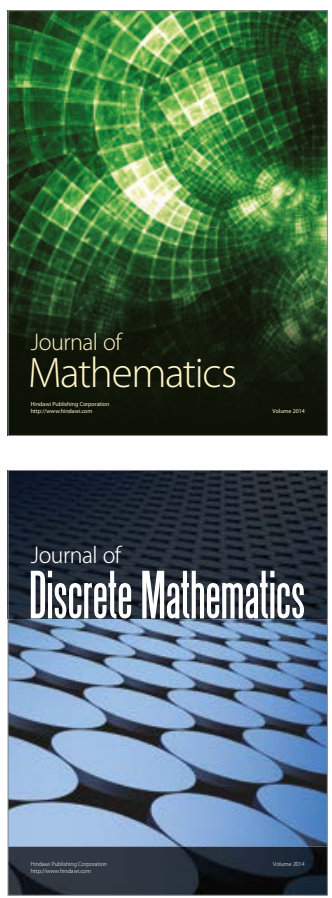

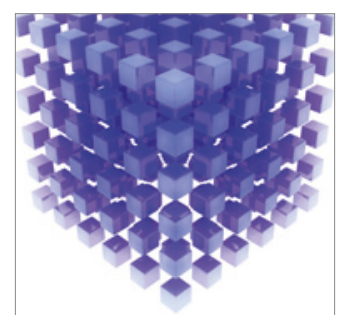

Mathematical Problems in Engineering
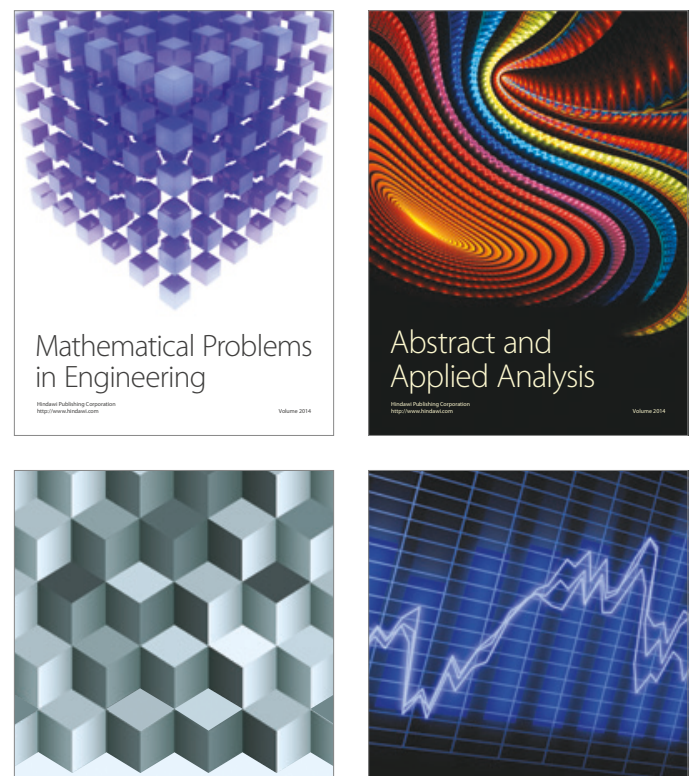

Journal of

Function Spaces

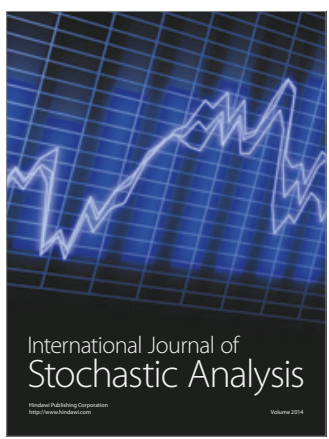

Probability and Statistics
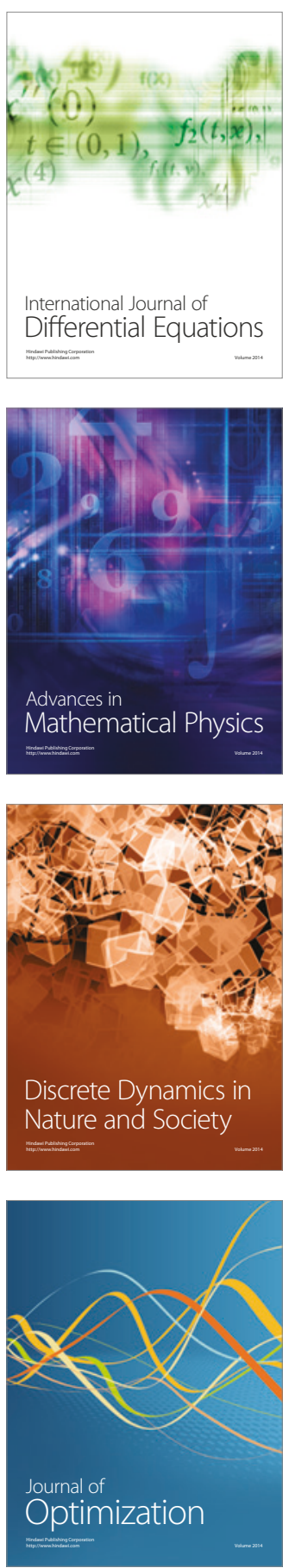